\title{
Electrostatic Asymmetry of Single-Crystal Nanostructures and Their Photocatalytic Properties
}

Ming Zhou

University of Michigan

Ji-Young Kim

University of Michigan

Myung Geun Han

Brookhaven National Laboratory https://orcid.org/0000-0002-3736-3281

\section{Xiaotao Zu}

University of Electronic Science and Technology of China

\section{Kai Sun}

University of Michigan

\section{Sa Zhang}

University of Electronic Science and Technology of China

\section{Fengai Zhao}

University of Electronic Science and Technology of China

\section{Haiyan Xiao}

University of Electronic Science and Technology of China

\section{Xia Xiang}

University of Electronic Science and Technology of China

\section{Yimei Zhu}

Brookhaven National Laboratory

Nicholas A Kotov ( $\nabla$ kotov@umich.edu )

University of Michigan https://orcid.org/0000-0002-6864-5804

\section{Article}

Keywords: cadmium sulfide, transmission electron microscopy, self-assembly, nanoscale asymmetry, nanoplatelets, oriented attachment, non-traditional crystallization

Posted Date: December 28th, 2020

DOI: https://doi.org/10.21203/rs.3.rs-123038/v1

License: (9) This work is licensed under a Creative Commons Attribution 4.0 International License. 



\section{Electrostatic Asymmetry of Single-Crystal Nanostructures and Their Photocatalytic Properties}

Ming Zhou, Ji-Young Kim*, Myung-Geun Han, Xiaotao Zu*, Kai Sun, Sa Zhang, Fengai Zhao, Haiyan Xiao, Xia Xiang, Yimei Zhu, * and Nicholas A. Kotov*

Dr. M. Zhou, Prof. X.T. Xiao, Dr. S. Zhang, Dr. F.A. Zhao, Prof. H.Y. Xiao, Prof. X. Xiang School of Physics, University of Electronic Science and Technology of China, Chengdu, 610054, PR China,

E-mail: xtzu@uestc.edu.cn

Dr. M. Zhou, Dr. J.--Y. Kim, Prof. N.A. Kotov

Department of Chemical Engineering, University of Michigan, Ann Arbor, Michigan 48109, USA

E-mail: jyoukim@umich.edu, kotov@umich.edu

Dr. K. Sun

Department of Materials Science and Engineering, University of Michigan, Ann Arbor, Michigan 48109, USA

Dr. M.-G. Han, Prof. Y. Zhu

Condensed Matter Physics and Materials Science, Brookhaven National Laboratory, Upton, NY 11973, USA

Email: zhu@bnl.gov

Prof. N.A. Kotov

Biointerfaces Institute University of Michigan, Ann Arbor, Michigan 48109, USA.

Keywords: cadmium sulfide, transmission electron microscopy, self-assembly, nanoscale asymmetry, nanoplatelets, oriented attachment, non-traditional crystallization

Abstract: Efficient light absorption and high energy of charge carriers of zinc cadmium sulfide (ZCS) make this semiconductor attractive for many photocatalytic reactions. Despite marked 
successes in shape-controlled synthesis of ZCS central to their photocatalytic performance, recombination of charge carriers as they migrate through the nanoscale particles result in losses of excitation energy markedly reducing photocatalytic activity of ZCS and other heterogeneous photocatalysts. Here we show that electrostatic asymmetry of single-crystalline ZCS with planar geometry assists charge separation and substantially increase the yield of photocatalytic reactions. The synthesized ZCS nanorods and nanoplates with identical chemical composition were found to have markedly different photocatalytic activity for evolution of hydrogen in water. Despite much smaller specific surface areas, the $\sim 500 \mathrm{~nm}$ wide nanoplates displayed hydrogen evolution rate 12 times higher than the $\sim 35 \mathrm{~nm}$ long nanorods also outperforming other ZCS photocatalysts. Experimental and computational data indicate that the homo and heterojunction-free ZCS nanoplates with continuous wurtzite lattice behave essentially as nanoscale dipoles. Electric-field-directed migration of charge carriers stimulates their localization on opposite parts of the nanoplates. Direct imaging of intraparticle electrical field using off-axis electron holography confirmed their electrostatic asymmetry. Polarizationenhanced charge separation provides a new pathway to efficient and stable photocatalysts for sustainable energy technologies.

Keywords: nanoscale asymmetry, catalysis, dipole, electron holography, self-assembly,

\section{Introduction}

Finding photonically efficient and chemically robust catalysts for hydrogen production is essential for sustainability of future economies. ${ }^{[1]}$ Intense light absorption and high conduction band energy make semiconductor alloy $\mathrm{Z}_{\mathrm{x}} \mathrm{Cd}_{1-\mathrm{x}} \mathrm{S}$ (ZCS) a potent photocatalysts for reduction of water into hydrogen. ${ }^{[2]}$ However, the rapid recombination of photogenerated carriers and related severe photocorrosion are regarded as unavoidable problems for ZCS nanostructures. Efforts to mitigate these bottlenecks were primarily focused on forming 
heterojunctions of nanoscale ZCS with metals, ${ }^{[3]}$ nanocarbons, ${ }^{[4]} \mathrm{Cu}_{\mathrm{x}} \mathrm{S},{ }^{[5]} \mathrm{MoO}_{2},{ }^{[6]} \mathrm{PdS},{ }^{[7]}$ $\mathrm{WS}_{2},{ }^{[8]} \mathrm{CoP},{ }^{[9]} \mathrm{Ni}_{2} \mathrm{P},{ }^{[10]} \mathrm{NiS},{ }^{[11]} \mathrm{TiO}_{2},{ }^{[12]} \mathrm{C}_{3} \mathrm{~N}_{4},{ }^{[13]} \mathrm{ZnO},{ }^{[14]}$ and other compounds. ${ }^{[15]}$ A variety of photocatalysts with homojunctions where charge separation is assisted by interfaces between the zinc-blende (ZB) and wurtzite (WZ) crystal phases of $\mathrm{ZCS}^{[16,17]}$ and combinations of homoand heterojunctions with co-catalysts ${ }^{[18,19]}$ were also tested. Overall, higher photocatalytic activity (PA) was found for homojunctions than for comparable heterojunctions because the high-contrast interfaces increase the barrier for charge transport and electron scattering; the junctions between dissimilar materials also enhance photocorrosion.

Albeit using largely Edissonian methods, previous studies on $\mathrm{CdS}$ give important clues about preferential nanoscale geometry for photocatalytic water splitting. The rods and nanoplates (NPLs) with $\{001\}$ facets serving as the primary catalytic plane displayed PA higher than the near-spherical CdS. ${ }^{[20-22]}$ Nanosheets and NPLs with dominant $\{001\}$ facets will be, thus, the geometry of choice for the ZCS photocatalysts. Two dimensional single-crystalline ZCS nanostructureswith dominant $\{001\}$ facets are still hard to make, however. Bin Zhang and co-workers reported a ZCS nanosheet prepared by cation-exchange reaction, but without dominant $\{001\}$ facets. Multistage synthesis can cause de-alloying of ZCS into ZnS and CdS phases destroying photophysical and chemical advantages of this alloy semiconductor. 
Nanostructures with continuous crystallinity and intrinsic static electric field would represent a nearly ideal case for efficient carrier separation in photocatalysts. ${ }^{[23]}$ The discovery of multiple nanostructures with electrostatic asymmetry ${ }^{[24-31]}$ opens a conceptual possibility to enhance the charge separation without adding the charge transport barriers. Here, we show that such possibility can be realized and utilized for monocrystalline ZCS with planar geometries. We synthesized ZCS NPLs and nanorods (NRs) with identical chemical composition and evaluated their PA in hydrogen evolution from water. The flat 'tops' and 'bottoms' of the NPLs with continuous wurtzite single-crystal lattice are formed by $\{001\}$ facets. The $\{001\}$ facets in wurtzite crystal lattice are asymmetric with one terminated with $\mathrm{Zn} / \mathrm{Cd}$ atoms and the opposite plane terminated with $\mathrm{S}$ atoms. ${ }^{[26]}$ The facet with an excess of metal atoms carries a positive charge, while the facet with an excess of sulfur atoms carries a negative charge. This intrinsic structural asymmetry of nanoscale particles ${ }^{[26,29-31]}$ without any other structural engineering of the nanoparticles results in the polarization field in their interior that promotes electron hole separation and their directed migration toward the opposite interfaces, which we utilized here to drastically increase $\mathrm{H}_{2}$ generation.

\section{Result and Discussion}

\subsection{From Nanoparticle to Nanorods and Nanoplatelets.}


The $\mathrm{Zn}_{0.5} \mathrm{Cd} \mathrm{d}_{0.5} \mathrm{~S}$ NRs were prepared via a solvothermal growth at $160^{\circ} \mathrm{C}$. Scanning electron microscopy (SEM) images (Figure 1a and Figure S1, Supporting Information) and transmission electron microscopy (TEM) bright-field (BF) images (Figure S2, Supporting Information) show that the synthesized NRs are 3-6 nm in width and 30-40 $\mathrm{nm}$ in length. When the temperature is raised to $220{ }^{\circ} \mathrm{C}$, the products are dominated by NPLs (Figure 1b). The temperature $\left(>200{ }^{\circ} \mathrm{C}\right)$ and concentration of ethylenediamine $(\approx 40 \mathrm{~mL})$ are the key factors determining the formation of the NPLs. Pure NPLs are obtained after purification of the reaction mixture by centrifugation in ethanol (Figure S3 and S4, Supporting Information).

In order to elucidate the mechanism of the NR $\rightarrow$ NPL transition, TEM BF images of the reaction mixture for different times at $220{ }^{\circ} \mathrm{C}$ were taken. The dispersion consisted mainly of NPLs for the reaction time of $72 \mathrm{~h}$ (Figure S5, Supporting Information). The transition from NRs to NPLs proceeds via non-classical crystallization pathways ${ }^{[31-33]}$ with platelets of about 5 $\mathrm{nm}$ in diameter serving as intermediates (Figure 1d-g, scanning transmission electron microscopy (STEM) annular dark-field (ADF) images). High-resolution STEM bright-field (BF) images (Figure 1h-k) shows the NPLs connected by small platelet in the same orientation, and both the NPLs and small platelet show clear lattice spacing with characteristic distance of $0.34 \mathrm{~nm}$, which can be indexed to the $\{100\}$ planes of the WZ ZCS (PDF No. 89-2943). 
Therefore, the most likely mechanism for the observed reconstruction involves restacking the nanoplatelets in a process of oriented attachment ${ }^{[31,32]}$.

XPS spectra (Figure S6, Supporting Information) reveal that the NRs and the NPLs had identical chemical compositions of $\mathrm{Zn}, \mathrm{Cd}$, and $\mathrm{S}$ atoms with identical valence state. The $\mathrm{Zn}: \mathrm{Cd}$ ratio determined by inductively coupled plasma optical emission spectrometry (ICP-OES, Figure S7, Supporting Information) is $1: 1$; it is equal to the $\mathrm{Zn}$ :Cd ratio used for the synthesis of the NRs and the NPLs.

Importantly, high-resolution TEM (HRTEM) image (Figure 2a) shows that NRs are segmented into cubic $\mathrm{ZB}$ and hexagonal $\mathrm{WZ}$ phase. These homojunctions are useful for enhancing of charge separation, but they also represent a double-edge sword simultaneously promoting charge electron scattering, surface entrapment, and photocorrosion. The ZB phase constitutes $<8 \mathrm{vol} \%$ of the NRs matching the X-ray diffraction (XRD) patterns (Figure S8, Supporting Information). The formation of the segmented NRs with WZ-ZB homojunctions was confirmed by fast Fourier transform (FFT) analysis performed at different regions outlined with the patterns inserted as insets in Figure 2a.

Contrary to the NRs case, only WZ phase was found in the NPLs confirmed by selected area electron diffraction (SAED) (Figure 2c) and XRD (Figure S8, Supporting Information) 
with all the diffraction peaks indexed to the standard hexagonal $\mathrm{Zn}_{0.5} \mathrm{Cd}_{0.5} \mathrm{~S}$ phase (PDF No.

89-2943). The elemental maps (Figure 2d) show that $\mathrm{Zn}, \mathrm{Cd}$ and $\mathrm{S}$ are homogeneously distributed throughout the entire NPL. A high-angle annular dark-field scanning transmission electron microscopy (HAADF-STEM) image acquired with atomic resolution along the $<001>$ axis (Figure 2e) corresponds to the crystal structure of the WZ-ZCS (Figure 2f). The lattice spacing with characteristic distance of $0.201 \mathrm{~nm}$ is indexed to the (110) planes of the WZ ZCS (PDF No. 89-2943). All data are consistent with NPLs having continuous hexagonal lattice with dominant $\{001\}$ facets.

\subsection{Photocatalytic Evolution of Hydrogen.}

Homojunctions found in the NRs (Figure 2a) were reported to facilitate photo-generated charge separation, ${ }^{[16,17]}$ and, thus, the NRs were expected to show much higher PA than NPLs. This, however, was not the case. $\mathrm{H}_{2}$ production from aqueous $\mathrm{Na}_{2} \mathrm{~S} / \mathrm{Na}_{2} \mathrm{SO}_{3}$ solution under visible light illumination $(\lambda \geq 420 \mathrm{~nm}$ ) was substantially higher for the NPLs (Figure 3a and Figure S9, Supporting Information). Large difference in PA can be also observed by the naked eye: the NPLs produced a large amount of gas bubbles during the illumination, while $\mathrm{H}_{2}$ evolution in case of the NRs was sluggish (Movie S1, Supporting Information).

The great PA difference between the NPLs and the NRs, is particularly surprising 
considering that the NPLs have ca $4 \mathrm{x}$ smaller specific surface area than the NRs. The surface area per gram of the nanomaterial for the NRs and the NPLs is $206 \mathrm{~m}^{2} \cdot \mathrm{g}^{-1}$ and $52 \mathrm{~m}^{2} \cdot \mathrm{g}^{-1}$, respectively, as measured from Brunauer-Emmett-Teller (BET) adsorption-desorption isotherms (Figure 3b). The average pore size is $10 \mathrm{~nm}$ for NRs while they range from 20 to 70 $\mathrm{nm}$ for the NPLs. It should be noted that the average pore in our case refer to the gap between particles, the smaller average pore size indicates that the aggregation of NRs is more serious than NPLs, which is consistent as we observed in SEM images (Figure 1a). Nevertheless, the NRs still have larger BET surface than that of the NPLs.

The apparent quantum yields (AQY) of $\mathrm{H}_{2}$ generation for the NPLs were $8.7 \%$ and $5.8 \%$ at $435 \mathrm{~nm}$ and $450 \mathrm{~nm}$, respectively (Figure S10, Supporting Information). The highest $\mathrm{H}_{2}$ production rate for the NPLs was $671 \mu \mathrm{mol} \cdot \mathrm{h}^{-1} \cdot \mathrm{mg}^{-1}$, which is 12 times higher than that of the NRs $\left(55 \mu \mathrm{mol} \cdot \mathrm{h}^{-1} \cdot \mathrm{mg}^{-1}\right)$. It also exceeded numerous other nanostructures with comparable composition reported previously with $\mathrm{H}_{2}$ production rate for the NPLs surpassing ZCS catalysts modified with noble metals (Table S1, Supporting Information). What is also remarkable is that the NPLs maintain a high $\mathrm{H}_{2}$ production rate of $554 \mu \mathrm{mol} \cdot \mathrm{h}^{-1} \cdot \mathrm{mg}^{-1}$ even when the concentrations of $\mathrm{Na}_{2} \mathrm{~S}$ and $\mathrm{Na}_{2} \mathrm{SO}_{3}$ are as low as $0.05 \mathrm{M}$ (Figure 3c), which is in stark contrast with other II-VI photocatalysts. ${ }^{[34]}$ Furthermore, the dispersion of NPLs retained $67 \%$ of its 
catalytic activity after 102 hours of illumination highlighting high resistance of NPLs to photocorrosion (Figure 3d). It should be noted here that $67 \%$ is a conservative estimate considering that a small part of catalyst will inevitably be lost via the splashed bubble during each process of vacuum pumping.

\subsection{Optical Properties.}

We initially hypothesized that dramatically increase of PA for NPLs vs NRs is related to the difference in their optical properties, which can be visualized by the color change of the samples from light to dark yellow (Figure 4a). Absorption thresholds of the NRs and the NPLs are 495 and $583 \mathrm{~nm}$ (Figure 4b) with band gap energies, $E_{g}$, being $2.57 \mathrm{eV}$ and $2.26 \mathrm{eV}$ (Figure S11, Supporting Information), respectively. The narrower band gap of NPLs vs NRs could be attributed to an increase of the characteristic size of the NPLs nanocrystals, ${ }^{[35]}$ which can be confirmed from the Mott-Schottky plots and ultraviolet photoelectron spectra. The conduction band (CB) minima of the NPLs deduced from the Mott-Schottky plots was found to be $-0.76 \mathrm{~V}$, while that of NRs was -1.02 V (vs. SCE) (Figure S12a-b, Supporting Information). Although the CB energy of the NPLs is reduced by $\sim 0.26 \mathrm{~V}$ compared to the NRs, it remains, nevertheless, thermodynamically sufficient for water reduction into hydrogen. ${ }^{[36]}$ The valence band (VB) maxima deduced from ultraviolet photoelectron spectra (Figure S12c-d, Supporting 
Information) are 0.89 and $0.93 \mathrm{eV}$ for the NRs and the NPLs, respectively. Both of them are also sufficient for redox reaction with hole scavengers.

Looking further into the behavior of charge carriers, photoluminescence (PL) spectra were collected from both the NPLs and the NRs ethanol suspension. The band-edge emission (BE) is observed in $450 \mathrm{~nm}$ regions and is quite weak for both NPLs and NRs, which can be attributed to the fast electron-hole separation in both cases. Unlike the NPLs, the NRs exhibit a strong defect emission (DE) peak at $572 \mathrm{~nm},{ }^{[37]}$ (Figure 4g). The lack of defect emission for the NPLs is consistent with its crystal structure homogeneity.

Furthermore, when the dispersion solvent is replaced by water, aqueous solution of $\mathrm{NaOH}$ or $\mathrm{Na}_{2} \mathrm{~S} / \mathrm{Na}_{2} \mathrm{SO}_{3}$, the intensity of DE from the NRs strongly decreased (Figure $4 \mathbf{h}$ and Figure S13, Supporting Information), which is consistent with the fact that the PA of CdS NRs highly depends on concentration of hydroxyl ions and scavengers ${ }^{[38,39]}$. On the contrary, the emission intensity of the NPLs have almost no changes both at BE and DE regions when the solvent were changed, indicating the NPLs can maintain fast electron-hole separation in different concentration solvent, which is consistent with the NPLs can maintain a high PA at low concentrations of $\mathrm{Na}_{2} \mathrm{~S}$ and $\mathrm{Na}_{2} \mathrm{SO}_{3}$. 


\subsection{Electrostatic Asymmetry of the NPLs.}

Comparative measurements of photocurrents generated by films of the NRs and the NPLs deposited on fluorine doped tin oxide (FTO) glass were carried out to characterize how many photoelectrons can successfully reach the particle surface and to better understand the mechanism of high PA. The photocurrent response of the NRs is slow and weak, reaching the maximum of $0.18 \mu \mathrm{A} \cdot \mathrm{cm}^{-1}$ with a rise time of 20 seconds (Figure $5 \mathrm{a}$ and $\mathrm{b}$ ). Under the same light source, the NPLs exhibit a quick and intense photocurrent response with a maximum as high as $\sim 78 \mu \mathrm{A} \cdot \mathrm{cm}^{-1}$ and a rise time as short as one second. Notably, the photocurrent for the NPLs is 430 times higher than that for the NRs. This result indicates that the enhanced PA activity is due to the greatly improved charge separation in the NPLs. Considering $12 x$ difference in PA, this is unusual for two materials of the identical composition. Because the photocurrent usually underestimates the multiple of PA enhanced (Table S2, Supporting Information).

To understand better the reasons behind more efficient charge separation at the NPL/FTO than the NR/FTO interfaces, we proposed a mechanism in Figure 5. The NPLs and the NRs are oriented with their long axes parallel to the surface (Figure $\mathbf{5} \mathbf{c}$ and $\mathbf{d}$ ), which is reasonable as we can see from the SEM images that most of NPLs are oriented with their long axes parallel 
to the silicon wafer surface (Figure S3, Supporting Information), and as is common for nanocolloids with geometrical asymmetries. ${ }^{[40-42]}$ Note, however, that seemingly similar orientation has markedly different consequences for the two types of nanostructures. Owning to the asymmetry of the hexagonal crystal lattice, ${ }^{[43]}$ the $(001)$ and $(00 \overline{1})$ surfaces of ZCS are inequivalent: The (001) $\mathrm{Zn} / \mathrm{Cd}$-terminated plane is positively charged, whereas the $(00 \overline{1}) \mathrm{S}$ terminated plane is negatively charged, ${ }^{[26]}$ which induces an intrinsic polarization field along the [001] direction. The intrinsic dipole moment (DM) is primarily due to the ionic contribution amounting to $48.39 \mathrm{D}$ per unit cell, with its direction parallel to the [001] axis in the WZ lattice (Table 1). The small DM of $0.0881 \mathrm{D}$ per unit cell arising from the bond polarization is also parallel to the [001] direction, which is in agreement with semi-empirical calculations. ${ }^{[44,45]}$

The largest facets of the NPLs producing its 'top' and 'bottom' surfaces are $\{001\}$, while the NRs have a growth direction of [001]. So, the internal polarization field is perpendicular to the NPL/FTO interface but parallel to the NRs/FTO interface (Figure 5e and f). Therefore, the photo-generated electrons tend to move to (001) terminated surface, i.e., the 'top' and 'bottom' surface for the NPLs while the two tips for the NRs. Considering the roughness of the electrode surface, the polarized part NPLs with high density of charge carriers will always find contacts with electrodes (inserts in Figure 5c and d). Consequently, the photo-generated charges can be 
efficiently transferred from the NPLs to the FTO electrodes. Besides some geometrical disadvantage with charge transfer to electrode that could be small, the electrostatic asymmetry of NRs is substantially reduced by the presence of several ZB segments. When it is large enough to assist the charge separation, the intrinsic polarization drives the charges toward the ends of the NRs rather than toward FTO interface as for NPLs.

The direct confirmation of electrostatic asymmetry of NPLs was obtained by off-axis electron holography $(\mathrm{EH})$ that enables profiling electrostatic potential through the particle with nanometer-resolution (Figure 6). ${ }^{[46,47]}$ To examine the presence of the static potential gradient in NPLs, we embedded the NPLs in the carbon film on Si substrate and then cross-sectioned them by focused ion beam milling. The carbon film mitigates unwanted charging due to the screening of local electrostatic polarization of the NPLs and induced by electron-beam. The phase shift map of NPLs after thickness correction (details in Supporting Information, Figure S14) shows considerable asymmetry (Figure 6a). The line profile of phase shift $(\phi(x))$ along the direction of the short axis of the NPLs (Figure 6b) shows vivid increase of phase shift ( $\Delta$ $\phi(\mathrm{x})=0.4 \mathrm{rad}$ ) for the NPL region (between spots 2 and 3, Figure 6c). The phase gradient in this region is linearly fitted with a slope of $0.002 \mathrm{rad} / \mathrm{nm}$, which corresponds to electric field of $0.01 \mathrm{~V} / \mathrm{nm}$ pointing from the $\mathrm{Zn} / \mathrm{Cd}$-terminated plane (spot 3 ) to the S-terminated plane (the 
spot 2). The electrostatic asymmetry in NRs along their longitudinal axis were also directly observed by EH (Supporting Information, Figure S15). The set of EH data confirms that internal polarization field is perpendicular to the NPL/FTO interface but parallel to the NRs/FTO interface.

\section{Conclusions}

Single-crystal ZCS NPLs with dominant $\{001\}$ facets have been prepared via a solvothermal method. The intrinsic polarization and ability of NPLs to self-assemble at the interfaces dramatically enhance the charge separation efficiency leading to markedly enhanced photocurrent. These findings make possible a new structural design approach for highly efficient photocatalysts that can be extended to many photocatalytic systems and photocurrent devices requiring efficient charge separation and transport.

\section{Experimental}

Synthesis of $\mathbf{Z n}_{0.5} \mathbf{C d} d_{0.5}$ S: All raw materials and reagents in this work were analytical grade and used without further purification. In a typical synthesis, $\mathrm{Zn}_{0.5} \mathrm{Cd}_{0.5} \mathrm{~S}, 0.5 \mathrm{mmol}$ zinc acetate $\left(\mathrm{Zn}(\mathrm{Ac})_{2} \cdot 2 \mathrm{H}_{2} \mathrm{O}\right)$, and $0.5 \mathrm{mmol}$ cadmium acetate $\left(\mathrm{Cd}(\mathrm{Ac})_{2} \cdot 2 \mathrm{H}_{2} \mathrm{O}\right)$ were dissolved in $10 \mathrm{~mL}$ ethylene glycol (EG) to form a $\mathrm{Zn} / \mathrm{Cd}-\mathrm{EG}$ solution; $2 \mathrm{mmol} \mathrm{Na}_{2} \mathrm{~S} \cdot 9 \mathrm{H}_{2} \mathrm{O}$ was dissolved in 10 $\mathrm{mL}$ EG and then added slowly (drop by drop) into the $\mathrm{Zn} / \mathrm{Cd}-\mathrm{EG}$ solution under magnetic stirring. Then 10 40 mL ethylenediamine (EDA) was added into the above mixed solutions. The total volume of mixture was keep at $60 \mathrm{~mL}$ by adding EG. After successive vigorous stirring and ultrasonic treatment for $1 \mathrm{~h}$, the precursor was sealed in a $100 \mathrm{~mL}$ Teflon-lined stainless steel autoclave, then submitted to thermal treatment at $160-220^{\circ} \mathrm{C}$ for $48 \mathrm{~h}$. After the 
reaction was complete, the autoclave was naturally cooled down to room temperature. The final products were collected and washed alternately with deionized water and absolute ethanol several times using a centrifuge instrument, and then dried in a drying oven at $60{ }^{\circ} \mathrm{C}$ overnight. Characterization: The structure and phase purity of the as-synthesized $\mathrm{Zn} 0.5 \mathrm{Cd} 0.5 \mathrm{~S}$ samples were investigated by powder X-ray diffraction (XRD) with $\mathrm{Cu} \mathrm{K} \alpha$ radiation (DX-2700). SEM images were obtained by InspectF50 and FEI Helios 650 Nanolab SEM/FIB. Low-resolution TEM images were taken on a JEOL JEM-2010F operated at $200 \mathrm{keV}$, HRTEM and scanning transmission electron microscopy (STEM) data were obtained on a double Cs-corrected JEOL JEM-3100R05 STEM operated at $300 \mathrm{keV}$. Brunauer-Emmett-Teller (BET) specific surface areas were determined by nitrogen adsorption-desorption isotherm measurements at $77 \mathrm{~K}$ (ASAP 2020 HD88). X-ray photoelectron spectroscopy (XPS) data were collected on a Kratos Axis Ultra XPS photoelectron spectrometer ( $\mathrm{Al} K \alpha, h v=1486 e V$ ), and the XPS spectra were all calibrated to the $\mathrm{C} 1 \mathrm{~s}$ peak at $284.6 \mathrm{eV}$. The elemental compositions of samples were determined by inductively coupled plasma optical emission spectroscopy on an Agilent 730 series ICP-OES. The ultraviolet-visible (UV-Vis) diffuse reflectance spectra were measured on a UV-visible spectrophotometer (UV3600) with integrating sphere attachment using $\mathrm{BaSO}_{4}$ as the reflectance sample. The photoluminescence (PL) spectra for water dispersed samples were investigated by use of a SHIMADZU RF-5301PC fluorescence spectrophotometer.

Photoelectrochemical measurements: Photoelectrochemical measurements were conducted in a conventional three-electrode cell system by using a CHI660E electrochemical station. The cleaned fluorine-doped tin oxide (FTO) glass deposited with samples, a Pt flake $(1 \mathrm{~cm} \times 1 \mathrm{~cm})$, and a saturated calomel electrode (SCE) were respectively used as working electrodes, counter electrode, and reference electrode. $0.1 \mathrm{M} \mathrm{Na}_{2} \mathrm{SO}_{4}$ was used as the electrolyte. A $300 \mathrm{~W}$ Xenon lamp with a UV cut-off filter $(\lambda \geq 420 \mathrm{~nm})$ was used as the light source. The working electrodes were prepared as follows: FTO glass were cleaned by ultrasonic treatment with ultrapure water, acetone, and absolute ethanol in sequence. $5 \mathrm{mg}$ of the as-prepared samples were dispersed in the mixed solution of $100 \mu \mathrm{L}$ Nafion and $1000 \mu \mathrm{L}$ absolute ethanol by ultrasonic treatment for about 5 min., then $100 \mu \mathrm{L}$ suspension were dropped onto the surface of a cleaned FTO plate and dried at room temperature. The photocurrent signals of the samples were measured under the $300 \mathrm{~W}$ Xenon lamp with a UV cut-off filter $(\lambda \geq 420 \mathrm{~nm})$ irradiation with light ON/OFF switches of $20 \mathrm{~s}$ at an applied potential of $0 \mathrm{~V}$ versus SCE. To obtain Mott-Schottky plots, two sweeps were conducted with an amplitude of $5 \mathrm{mV}$ at the frequencies of 1 and $2 \mathrm{kHz}$.

Computational Details: Density function theory (DFT) calculations were performed using the 
Vienna ab initio simulation package (VASP) with the exchange-correction effects treated by generalized gradient approximation (GGA). The interactions between ions and electrons were described by the projector augmented-wave (PAW) method. A $4 \times 2 \times 2$ supercell containing 64 atoms were used. A cutoff energy of $450 \mathrm{eV}$ for the plane-wave basis set. The dipole moments were calculated using the Berry phase method.

Electrostatic potential gradient analysis: The sample for off-axis electron holography were prepared via FIB (focused ion beam) lift-out technique on FEI Helios 650 Nanolab SEM/FIB as follows: the NPLs dispersions were put on a clean silicon wafer, then a layer of carbon and platinum were successively plated on the surface of NPLs for protect the NPLs from damage during FIB lift-out process.

Off-axis electron holograms of NPLs were acquired using the JEOL 2100F Lorentz microscope operated at $200 \mathrm{kV}$ equipped with a biprism. The phase images were reconstructed using custom Gatan Digital Micrograph scripts (source code is available on request). The phase profile was calculated in Gatan Digital Micrograph using the line profile tool. The electrostatic potential gradient was determined by evaluating the phase shift, $\phi(x)$, of an electron wave passing through the specimen in the line profile, relative to the wave travelling through a vacuum:

$\phi(x)=\mathrm{C}_{E} \int\left\{V_{M I P}(x z)+V_{C P}(\mathrm{x} \mathrm{z})\right\} \mathrm{dz}$

where $\mathrm{z}$ is the incident beam direction, $\mathrm{x}$ is an in-plane direction of the sample, $V_{M I P}$ is the mean inner potential, $V_{C P}$ is the potential from charge accumulation and $\mathrm{C}_{\mathrm{E}}$ is the interaction constant depending on the acceleration voltage of the electron microscope $\left(\mathrm{C}_{\mathrm{E}}\right.$ of a $200 \mathrm{kV}$ electron beam $=0.00729 \mathrm{rad} / \mathrm{V} \cdot \mathrm{nm}) . V_{M I P}$ is constant for any given position for ZCS NPLs since they are homogeneous material. Assuming no fringing electric fields outside the TEM sample, the phase shift $\phi(\mathrm{x})$ is simplified as the following:

$$
\phi(x)=\mathrm{C}_{E}\left\{V_{M I P}(x)+V_{C P}(\mathrm{x})\right\} \mathrm{t}(\mathrm{x})
$$

where $t(x)$ is the local TEM sample thickness.

Finally, the $\mathrm{V}_{\mathrm{CP}}(\mathrm{x})$ can be obtained by rearranging the Eq. 2:

$$
V_{C P}(\mathrm{x})=\frac{\mathrm{C}_{E} \phi(x)}{\mathrm{t}(\mathrm{x})}-V_{M I P}(x)
$$


High-angle annular dark-field (HAADF)-STEM has been performed to evaluate the thickness $t(x)$ of the same sample using JEOL ARM 200CF microscope equipped with a cold field-emission gun and spherical aberration corrector operated at $200 \mathrm{kV}$ (Figure S12). Since the intensity of HAADF signal for same material is proportional to $t$, the HAADF signal in each material region show the tendency of thickness variation for each region. The linear fitted slopes of three main parts, silicon wafer, ZCS NPL, and platinum are $-0.023,-0.036$, and $-0.088 \mathrm{~nm}^{-1}$, respectively (Figure 12c). This is typical t variation in cross-section TEM samples prepared by FIB, of which $t(x)$ decreases as close to the start point of milling process (platinum layer) (Figure S12d) However, the $\Delta \phi$ showed different trend between the silicon and the NPL: $\Delta \phi_{\text {Silicon }}(-0.0090 \mathrm{rad} / \mathrm{nm})<\Delta \phi_{N P L}(-0.0062 \mathrm{rad} / \mathrm{nm})$ (Figure S12e).

In order to remove the thickness variation effect and extract the $\mathrm{V}_{\mathrm{CP}}(\mathrm{x})$ from the phase shift map (Figure S12b), we flattened the phase shift slope in silicon wafer region (Figure S5, note the slope for silicon: $0 \mathrm{rad} / \mathrm{nm})$. This flattening process assumes a linear thickness variation $\mathrm{t}(\mathrm{x})$ for both the silicon and NPL region, solely leaving the $\mathrm{V}_{\mathrm{CP}}(\mathrm{x})$ with an offset of the local mean inner potential $\mathrm{V}_{\mathrm{MIP}}(\mathrm{x})$ in the Eq. 3. Note that the nonlinear thickness variation of platinum layer is not completely corrected (as shown negative slope in Fig. 6c for the region) because we did "flattening" with respect to silicon, of which thickness variation is linear. After this flattening process, if we assume that NPL has no electric field, we can expect the flat phaseshift from NPL as the black dashed line in Fig. 6c. However, the slope in NPL increases, which opposes to the phase shift variation caused by thickness reduction, which represents that this increasement can be only caused by charge potential and it could be even slightly underestimated due to imperfect thickness correction. The offset correction was also made by setting vacuum to zero, corresponding to zero phase shift. There was no noticeable phase shift that present a space charge region near the Si edge, otherwise showing a quadratic change in the phase shift. The edges of NPL region shown in phase shift (Figure 6c and Figure S12e) profiles are not sharp because we made line profile with integration width of 20 pixels. Due to the electric field of NPL, the phase shift of the carbon region on platinum side (between lines 3 and 4) is higher than the phase shift of the carbon on silicon wafer side (between lines 1 an 2) (Figure 6c). In order to quantitatively measure the local sample thickness $t(x)$, electron energy loss spectroscopy (EELS) was performed using JEOL 3100R05 STEM operated at 300 $\mathrm{keV}$ for the same sample. By importing electron mean-free path $(133.5 \mathrm{~nm})$ and absolute thickness of specimen obtained by EELS, the phase shift gradient $(0.002 \mathrm{rad} / \mathrm{nm})$ has been converted into electrostatic potential gradient $(0.01 \mathrm{~V} / \mathrm{nm})$. 
Photocatalytic activity measurement: Photocatalytic $\mathrm{H}_{2}$ production experiments were carried out in a top-irradiation vessel connected to a closed gas-circulation system. In a typical run, 5 $\mathrm{mg}$ of the photocatalysts were dispersed by ultrasonic treatment in $20 \mathrm{~mL}$ deionized water and then $4 \mathrm{~mL}$ of samples $(1 \mathrm{mg}$ ) were pipetted into $100 \mathrm{~mL}$ of aqueous solution containing $\mathrm{Na} 2 \mathrm{~S}$ $(0.75 \mathrm{M})$ and $\mathrm{Na}_{2} \mathrm{SO}_{3}(1.05 \mathrm{M})$ as sacrificial reagents. After degassing the system for ten minutes to remove most of the dissolved oxygen, a 300W xenon lamp (16 A), equipped with a cut-off filter ( $\lambda \geq 420 \mathrm{~nm}$, Figure S14a), was applied to execute the photocatalytic reaction. The photocatalytic $\mathrm{H}_{2}$ evolution rate was analyzed using an GC-7806 gas chromatograph (GC, TCD detector, and Ar carrier).

The wavelength dependent apparent quantum efficiency (AQE) and stability was measured under similar photocatalytic reactions except the besides was wrapped by tin foil. 4 $\mathrm{mL}$ of samples $(1 \mathrm{mg})$ were pipetted into $100 \mathrm{~mL}$ of aqueous solution containing $\mathrm{Na}_{2} \mathrm{~S}(0.75$ $\mathrm{M})$ and $\mathrm{Na}_{2} \mathrm{SO}_{3}(1.05 \mathrm{M})$ as sacrificial reagents. The apparent quantum efficiency was measured using 435, 450, 475, and $500 \mathrm{~nm}$ band pass filter (Figure S14b). The QE was finally calculated according to the equation:

$$
\begin{aligned}
\operatorname{AQE}(\%) & =\frac{\text { number of reacted electrons }}{\text { number of incident photons }} \times 100 \% \\
& =\frac{\text { number of evolved } \mathrm{H} 2 \text { molecules } \times 2}{\text { number of incident photons }} \times 100 \%
\end{aligned}
$$

The power of the 435, 450, 475, and $500 \mathrm{~nm}$ light was $0.156,0.186,0.217$, and 0.175 $\mathrm{mW}$, corresponding to the number of incident photons of $3.414 \times 10^{17}, 4.210 \times 10^{17}, 5.185 \times$ $10^{17}$, and $4.402 \times 10^{17}$, respectively, which was measured by an irradiatometer (PM100D with Thermal Power Sensor Head, Surface Absorber, S310C).

\section{Supporting Information}

Supporting Information is available from the Wiley Online Library or from the author.

Acknowledgements: This work was supported by the NSAF Joint Foundation of China (U1830204 and U1630126), the China Scholarship Council (No. 201706070097), and the National Science Foundation under grant NSF 1463474, "Energy- and Cost-Efficient Manufacturing Employing Nanoparticles", NSF 1566460 Nanospiked Particles for Photocatalysis. We also give acknowledgements to the $\mathrm{MC}^{2}$ facility at the University of Michigan for assistance in acquisition of SEM and TEM images. The work at Brookhaven 
National Laboratory was supported by the Materials Science and Engineering Divisions, Office of Basic Energy Sciences of the U.S. Department of Energy under Contract No. DESC 0012704.

Author contributions: N.A.K. and X.-T.Z. conceived the project. M.Z. designed the study, prepared the samples to be characterized, performed the experiments. J.-Y. K, M.-G. H, and Y.Z. acquired electron microscopy images for asymmetry of electrostatic potential analysis. J.-Y. K. and M.-G. H. analyze the off-axis electron holography. M.Z. and K.S. acquired the SEM and TEM images. F.-A.Z., X.X., S.Z., and X.-Y.X. calculate the intrinsic dipole moments. N.A.K., K.S., M.Z., and J.-Y. K. wrote the manuscript.

\section{References}

[1] X. Chen, S. Shen, L. Guo, S. S. Mao, Chem. Rev. 2010, 110, 6503.

[2] Q. Li, H. Meng, P. Zhou, Y. Zheng, J. Wang, J. Yu, J. Gong, ACS Catal. 2013, 3, 882.

[3] S. R. Lingampalli, U. K. Gautam, C. N. R. Rao, Energy Environ. Sci. 2013, 6, 3589.

[4] J. Zhang, J. Yu, M. Jaroniec, J. R. Gong, Nano Lett. 2012, 12, 4584.

[5] Y. Chen, S. Zhao, X. Wang, Q. Peng, R. Lin, Y. Wang, R. Shen, X. Cao, L. Zhang, G. Zhou, J. Li, A. Xia, Y. Li, J. Am. Chem. Soc. 2016, 138, 4286.

[6] H. Du, X. Xie, Q. Zhu, L. Lin, Y. F. Jiang, Z. K. Yang, X. Zhou, A. W. Xu, Nanoscale 2015, 7, 5752 .

[7] K. Khan, X. Tao, M. Shi, B. Zeng, Z. Feng, C. Li, R. Li, Adv. Funct. Mater. 2020, 30, 2003731.

[8] H. Zhao, R. Sun, X. Li, X. Sun, Mater. Sci. Semicond. Process. 2017, 59, 68.

[9] B. Qiu, Q. Zhu, M. Xing, J. Zhang, Chem. Commun. 2017, 53, 897.

[10] L. Ye, C. Han, Z. Ma, Y. Leng, J. Li, X. Ji, D. Bi, H. Xie, Z. Huang, Chem. Eng. J. 2017, 307, 311.

[11] J. Zhang, L. Qi, J. Ran, J. Yu, S. Z. Qiao, Adv. Energy Mater. 2014, 4, 1301925. 
[12] A. Roy, S. R. Lingampalli, S. Saha, C. N. R. Rao, Chem. Phys. Lett. 2015, 637, 137.

[13] L. Yao, D. Wei, Y. Ni, D. Yan, C. Hu, Nano Energy 2016, 26, 248.

[14] S. R. Lingampalli, U. K. Gautam, C. N. R. Rao, Energy Environ. Sci. 2013, 6, 3589.

[15] J. Ran, B. Zhu, S. Z. Qiao, Angew. Chemie - Int. Ed. 2017, 56, 10373.

[16] M. Liu, D. Jing, Z. Zhou, L. Guo, Nat. Commun. 2013, 4, 1.

[17] M. Liu, L. Wang, G. Lu, X. Yao, L. Guo, Energy Environ. Sci. 2011, 4, 1372.

[18] J. Song, H. Zhao, R. Sun, X. Li, D. Sun, Energy Environ. Sci. 2017, 10, 225.

[19] M. Liu, Y. Chen, J. Su, J. Shi, X. Wang, L. Guo, Nat. Energy 2016, $1,1$.

[20] P. Kalisman, Y. Nakibli, L. Amirav, Nano Lett. 2016, 16, 1776.

[21] X. Wang, M. Liu, Z. Zhou, L. Guo, J. Phys. Chem. C 2015, 119, 20555.

[22] C. Li, L. Han, R. Liu, H. Li, S. Zhang, G. Zhang, J. Mater. Chem. 2012, 22, 23815.

[23] A. I. Hochbaum, P. Yang, Chem. Rev. 2010, 110, 527.

[24] F. Bernardini, V. Fiorentini, D. Vanderbilt, Phys. Rev. B - Condens. Matter Mater. Phys. 1997, 56, R10024.

[25] F. Bernardini, V. Fiorentini, Phys. Rev. B - Condens. Matter Mater. Phys. 1998, 57, R9427.

[26] M. Shim, P. Guyot-Sionnest, J. Chem. Phys. 1999, 111, 6955.

[27] M.-B. Lien, J.-Y. Kim, M.-G. Han, Y.-C. Chang, Y.-C. Chang, H. J. Ferguson, Y. Zhu, A. A. Herzing, J. C. Schotland, N. A. Kotov, T. B. Norris, ACS Nano 2017, acsnano.7b01665.

[28] J.-Y. Kim, M.-G. Han, M.-B. Lien, S. Magonov, Y. Zhu, H. George, T. B. Norris, N. A. Kotov, Sci. Adv. 2018, 4, e1700682.

[29] W. Feng, J.-Y. Kim, X. Wang, H. A. Calcaterra, Z. Qu, L. Meshi, N. A. Kotov, Sci. Adv. 2017, 3, e1601159. 
[30] Z. Tang, Z. Zhang, Y. Wang, S. C. Glotzer, N. A. Kotov, Science (80-. ). 2006, 314, 274.

[31] Z. Tang, N. A. Kotov, M. Giersig, Science (80-. ). 2002, 297, 237.

[32] J. J. De Yoreo, P. U. P. A. Gilbert, N. A. J. M. Sommerdijk, R. L. Penn, S. Whitelam, D. Joester, H. Zhang, J. D. Rimer, A. Navrotsky, J. F. Banfield, A. F. Wallace, F. M. Michel, F. C. Meldrum, H. Cölfen, P. M. Dove, Science (80-. ). 2015, 349.

[33] R. L. Penn, J. F. Banfield, Geochim. Cosmochim. Acta 1999, 63, 1549.

[34] Z. Sun, H. Zheng, J. Li, P. Du, Energy Environ. Sci. 2015, 8, 2668.

[35] Y. Li, M. Ye, C. Yang, X. Li, Y. Li, Adv. Funct. Mater. 2005, 15, 433.

[36] X. Y. Liu, H. Chen, R. Wang, Y. Shang, Q. Zhang, W. Li, G. Zhang, J. Su, C. T. Dinh, F. P. G. de Arquer, J. Li, J. Jiang, Q. Mi, R. Si, X. Li, Y. Sun, Y. T. Long, H. Tian, E. H. Sargent, Z. Ning, Adv. Mater. 2017, 29, 1.

[37] H. Li, X. Wang, J. Xu, Q. Zhang, Y. Bando, D. Golberg, Y. Ma, T. Zhai, Adv. Mater. 2013, 25, 3017.

[38] T. Simon, N. Bouchonville, M. J. Berr, A. Vaneski, A. Adrovic, D. Volbers, R. Wyrwich, M. Döblinger, A. S. Susha, A. L. Rogach, F. Jäckel, J. K. Stolarczyk, J. Feldmann, Nat. Mater. 2014, 13, 1.

[39] Z. Sun, H. Zheng, J. Li, P. Du, Energy Environ. Sci. 2015, 8, 2668.

[40] N. A. Kotov, Europhys. Lett. 2017, 119, 66008.

[41] L. Qu, Y. Liu, J.-B. Baek, L. Dai, ACS Nano 2010, 4, 1321.

[42] N. A. Kotov, I. Dékány, J. H. Fendler, 1995, 99, 13065.

[43] X. Li, Z. Li, J. Yang, Phys. Rev. Lett. 2014, 112, 1.

[44] M. Yang, K. Sun, N. A. Kotov, J. Am. Chem. Soc. 2010, 132, 1860.

[45] G. D. Lilly, J. Lee, K. Sun, Z. Tang, K. S. Kim, N. A. Kotov, J. Phys. Chem. C 2008, 112,370 . 
[46] M. J. Polking, M.-G. Han, A. Yourdkhani, V. Petkov, C. F. Kisielowski, V. V. Volkov, Y. Zhu, G. Caruntu, A Paul Alivisatos, R. Ramesh, Nat. Mater. 2012, 11, 700.

[47] E. Volk1, L. F. Allard, D. C. Joy, Introduction to electron holography; 1999.

[48] J. Zhang, M. Zhang, C. Yang, X. Wang, Adv. Mater. 2014, 26, 4121.

[49] K. Li, M. Han, R. Chen, S. L. Li, S. L. Xie, C. Mao, X. Bu, X. L. Cao, L. Z. Dong, P. Feng, Y. Q. Lan, Adv. Mater. 2016, 28, 8906.

[50] H. Huang, B. Dai, W. Wang, C. Lu, J. Kou, Y. Ni, L. Wang, Z. Xu, Nano Lett. 2017, $17,3803$.

[51] D. Li, S. H. Yu, H. L. Jiang, Adv. Mater. 2018, 30, 1707377.

[52] W. Che, W. Cheng, T. Yao, F. Tang, W. Liu, H. Su, Y. Huang, Q. Liu, J. Liu, F. Hu, Z. Pan, Z. Sun, S. Wei, J. Am. Chem. Soc. 2017, 139, 3021.

[53] Y. Li, R. Jin, Y. Xing, J. Li, S. Song, X. Liu, M. Li, R. Jin, Adv. Energy Mater. 2016, 6,1601273 .

[54] Y. Zhang, L. Wu, X. Zhao, Y. Zhao, H. Tan, X. Zhao, Y. Ma, Z. Zhao, S. Song, Y. Wang, Y. Li, Adv. Energy Mater. 2018, 8, 1801139.

[55] G. Zhang, L. Lin, G. Li, Y. Zhang, A. Savateev, S. Zafeiratos, X. Wang, M. Antonietti, Angew. Chemie Int. Ed. 2018, 9372.

[56] J. Ran, G. Gao, F.-T. Li, T.-Y. Ma, A. Du, S.-Z. Qiao, Nat. Commun. 2017, 8, 13907.

[57] Y. Yu, W. Yan, X. Wang, P. Li, W. Gao, H. Zou, S. Wu, K. Ding, Adv. Mater. 2018, $30,1705060$.

[58] K. Zhang, J. K. Kim, B. Park, S. Qian, X. Sheng, H. Zeng, S. H. Oh, C.-L. Lee, J. H. Park, Nano Lett. 2017, 17, 11, 6676-6683

[59] S. Zhang, X. Liu, C. Liu, S. Luo, L. Wang, T. Cai, Y. Zeng, J. Yuan, W. Dong, Y. Pei, Y. Liu, ACS Nano 2018, 12, 1, 751-758

[60] C. Zeng, Y. Hu, T. Zhang, F. Dong, Y. Zhang, H. Huang, J. Mater. Chem. A 2018. 6, 16932-16942 



\section{Figures}

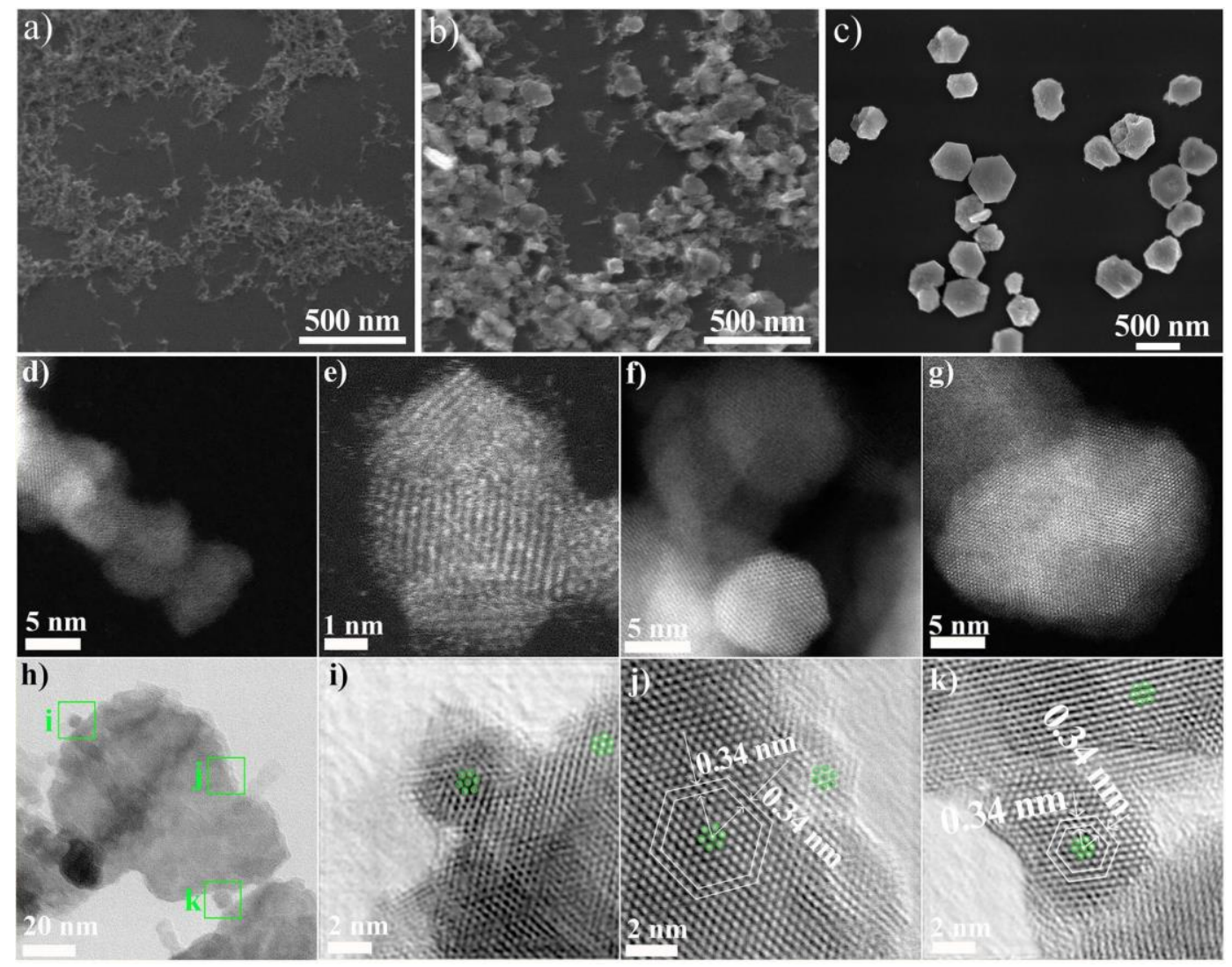

Figure 1. SEM images of the ZCS nanomaterial prepared at: a) $160^{\circ} \mathrm{C}$ for $48 \mathrm{~h}$; b) $220{ }^{\circ} \mathrm{C}$ for $48 \mathrm{~h}$; c) Pure NPLs are obtained after purification. STEM ADF (d-g) and (h-k) BF images of the ZCS prepared at $220^{\circ} \mathrm{C}$ for $48 \mathrm{~h}$. 


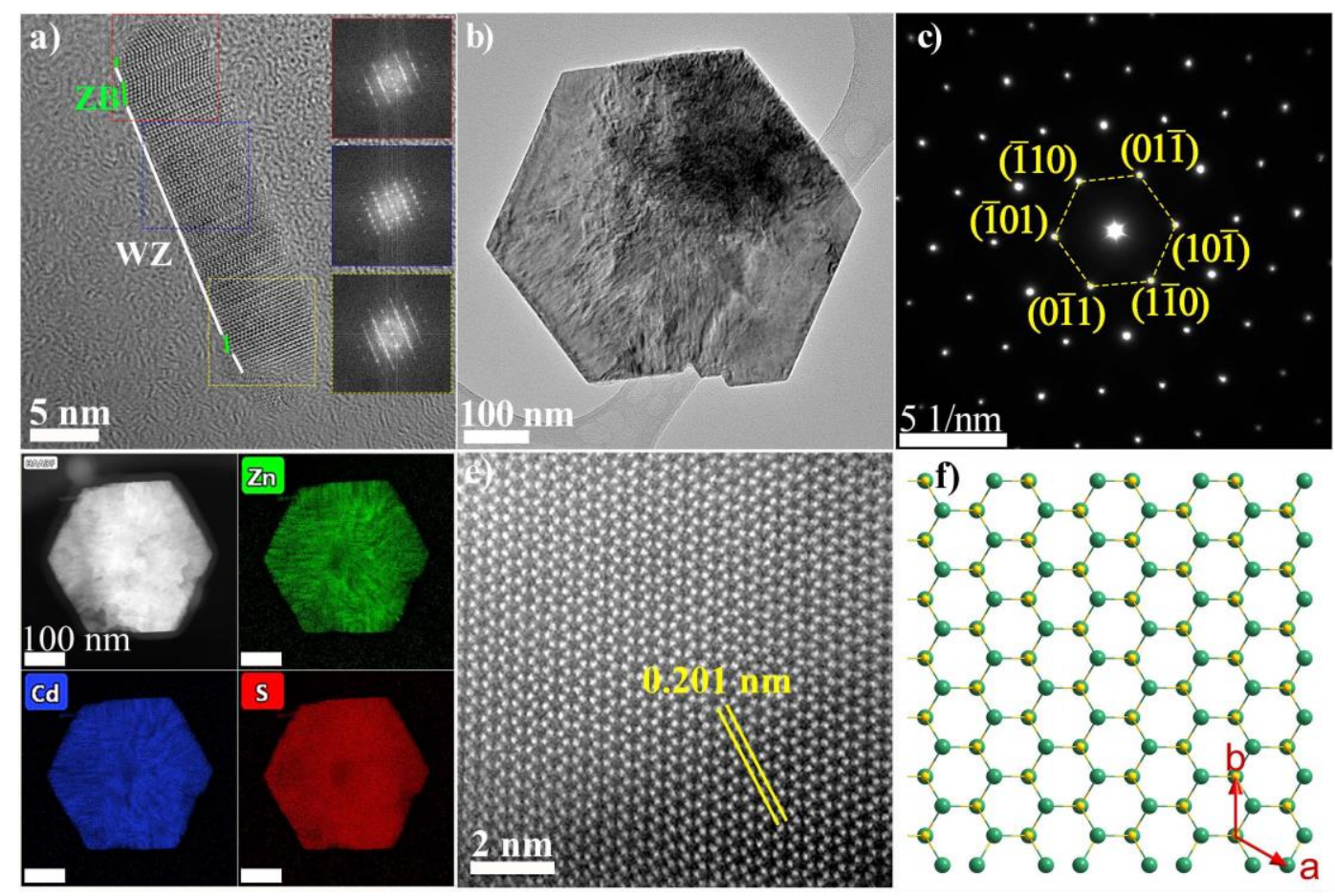

Figure 2. a) HRTEM images and FFT patterns of the ZCS NRs; b) TEM BF images of a ZCS NPLs; c) SAED pattern from the NPL in b); d) elemental mapping of the NPLs; e) Atomic resolution HAADF-STEM image along the [001] axis the NPLs. f) Atomic structure of the (001) plane of WZ-ZCS (green indicates $\mathrm{Cd} / \mathrm{Zn}$ atom, yellow indicates $\mathrm{S}$ atom). 

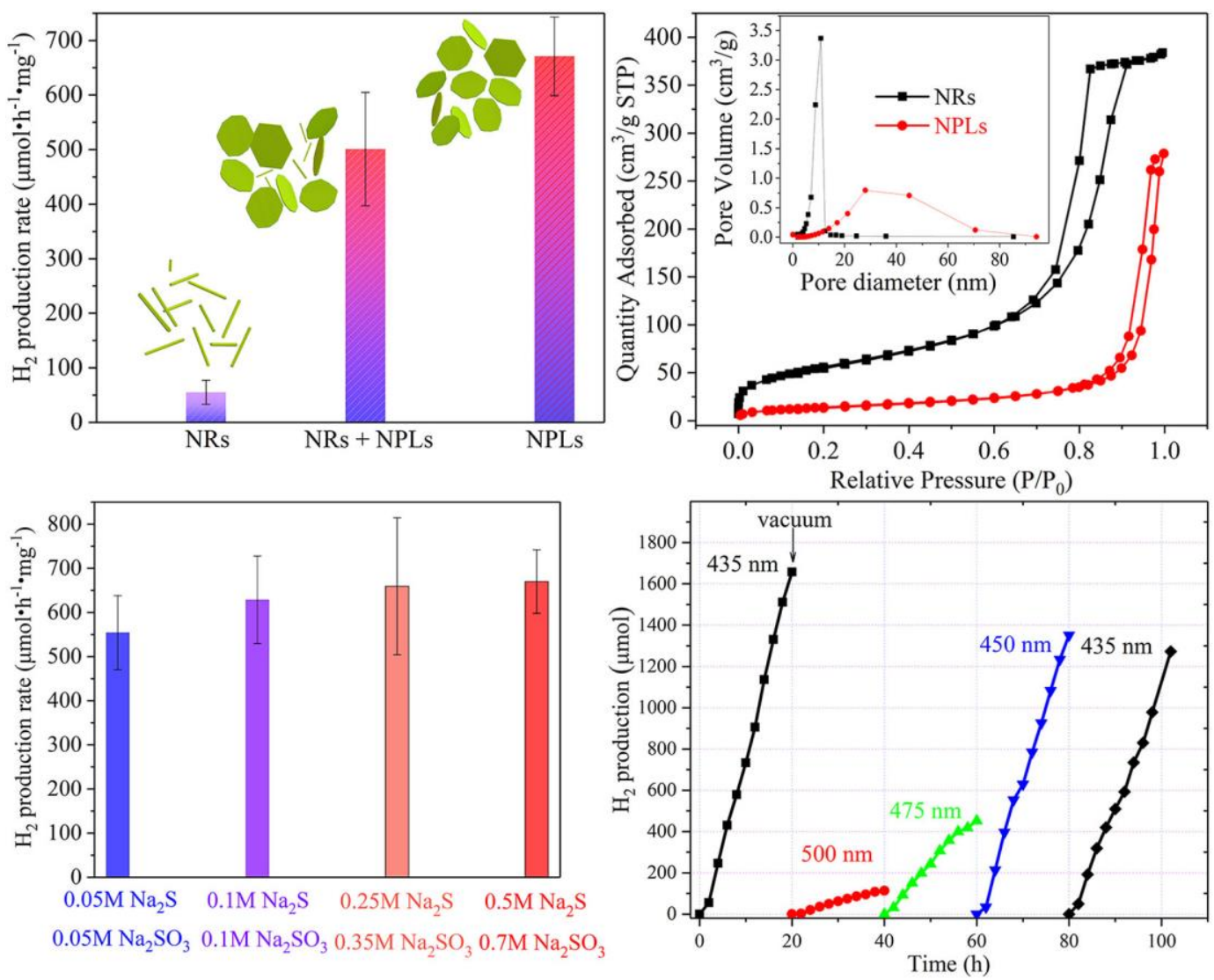

Figure 3. a) Photocatalytic $\mathrm{H}_{2}$ production rate for ZCS catalysts with different geometry: 1.0 mg of photocatalyst, $0.7 \mathrm{M} \mathrm{Na}_{2} \mathrm{~S}$, and $1.05 \mathrm{M} \mathrm{Na}_{2} \mathrm{SO}_{3}$; b) Brunauer-Emmett-Teller adsorptiondesorption isotherms of the NRs and the NPLs, pore size distribution by BJH in the insert; c) $\mathrm{H}_{2}$ production rate of the NPLs at different concentrations of hole scavengers. d) Cycling tests of photocatalytic activity of the NPLs under different wavelengths. Error bars in (b) and (c) represent standard deviation of measured rates within 3 hours. 

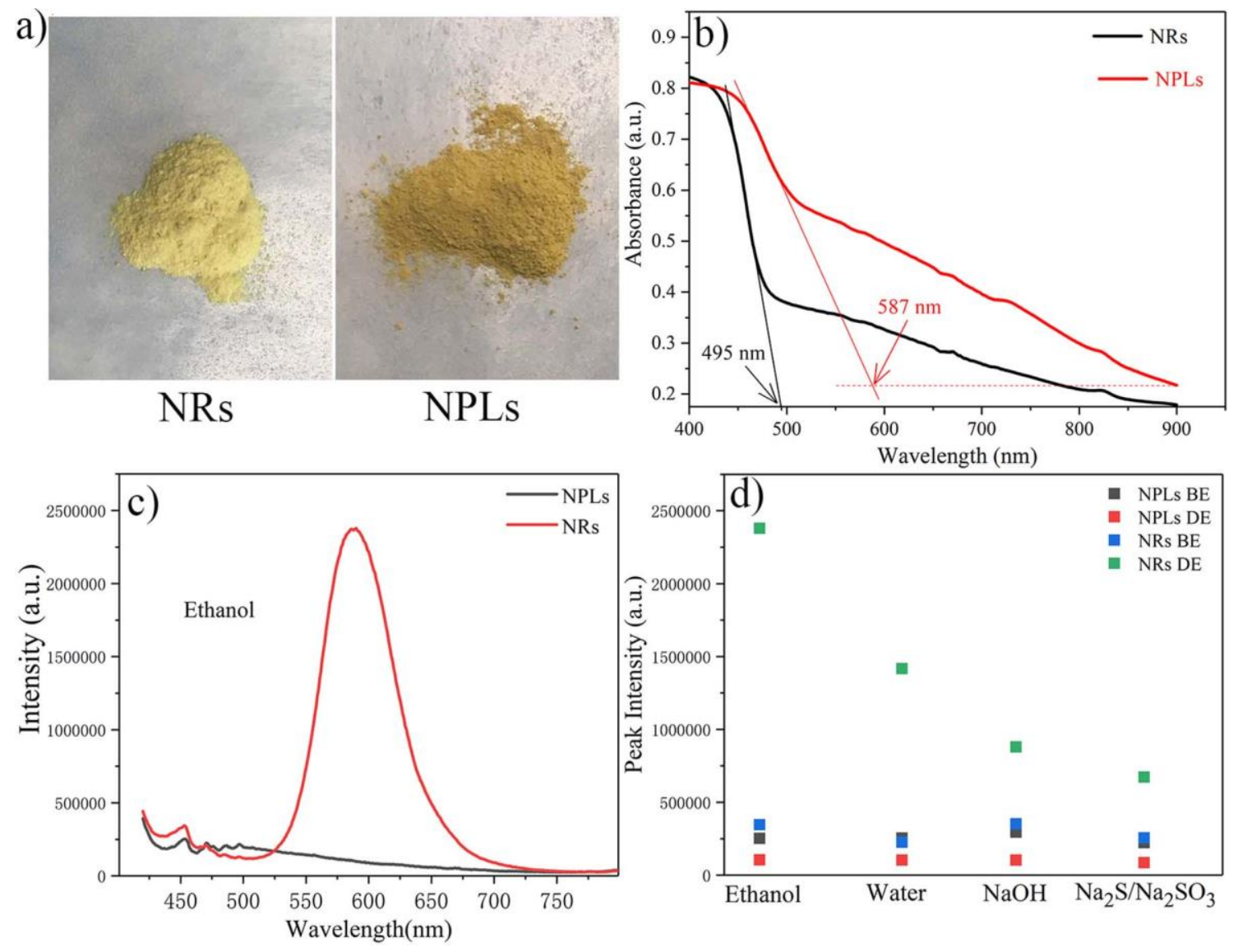

Figure 4. a) Photographs of the NRs and the NPLs samples used in photocatalysis experiments; b) UV-Vis spectra of the NRs and the NPLs, the inserts is the corresponding plots of $(a h v)^{2}$ versus the energy of light (hv); Mott-Schottky plots of the NRs (c) and NPLs (d); Ultraviolet photoelectron spectra of the NRs (e) and NPLs (f); g)Photoluminescence spectra of NPLs and NRs ( $1 \mathrm{mg} / \mathrm{mL})$ in ethanol; h) emission intensities in in ethanol, water, $\mathrm{NaOH}(10 \mathrm{M})$, and $\mathrm{Na}_{2} \mathrm{~S}$ $(0.7 \mathrm{M}) / \mathrm{Na}_{2} \mathrm{SO}_{3}(1.05 \mathrm{M})$ solutions. The excitation wavelength was $400 \mathrm{~nm}$. 

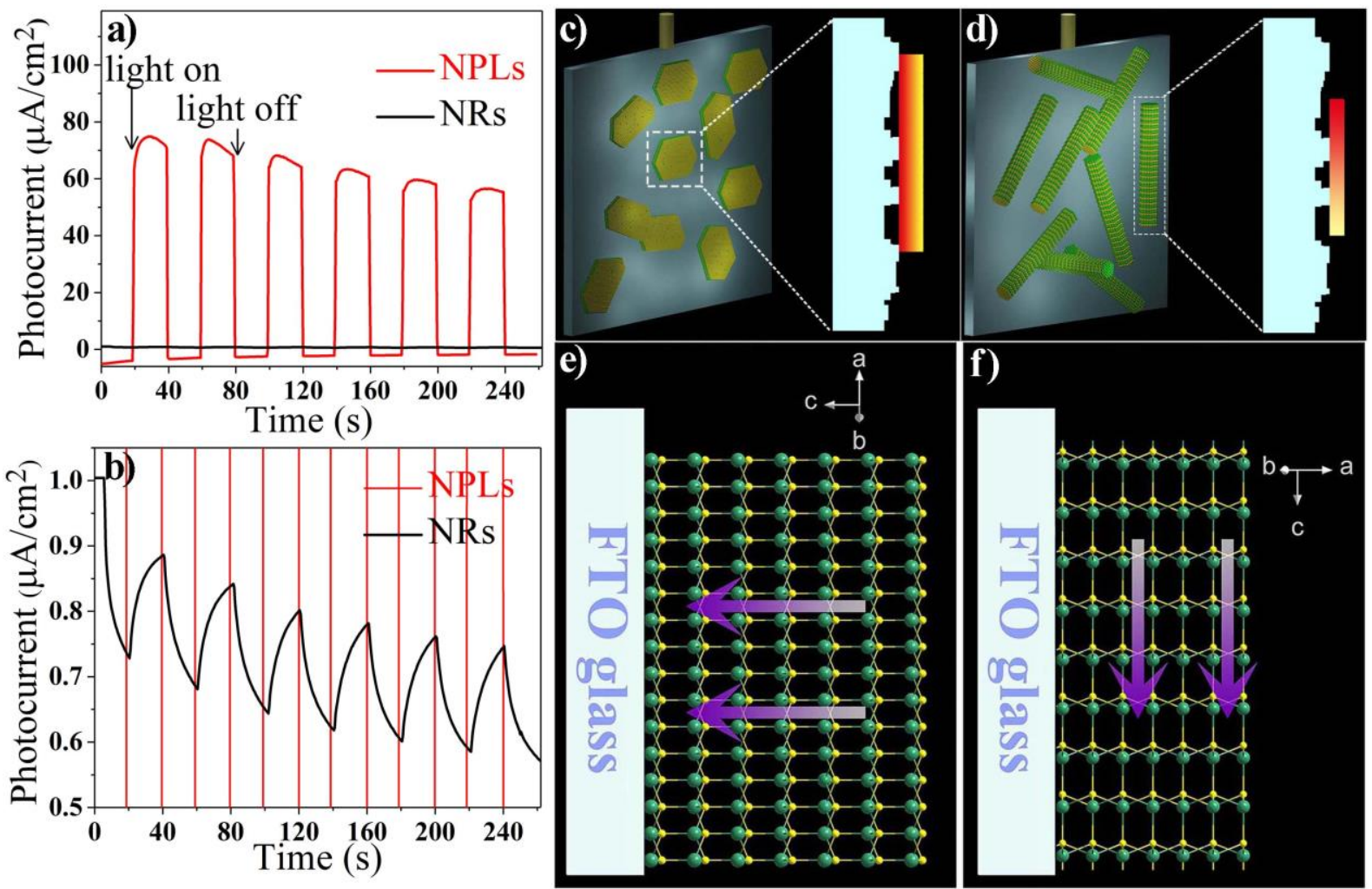

Figure 5. Photocurrent response (a) and locally enlarged photocurrent response (b) of the NRs and the NPLs. Schematic illustration of working electrode: (c) the NPLs and (d) the NRs, the inserts show the side view of electrodes. The color gradient indicates the charge gradient in the nanostructure. Schematic illustration of the intrinsic polarization field along the [001] direction for (e) the NPLs and (f) the NRs adsorbed to an FTO electrode.

Table 1 Calculated Dipole Moment (DM) of $\mathrm{Zn}_{0.5} \mathrm{Cd}_{0.5} \mathrm{~S}$ lattice.

\begin{tabular}{lccc}
\hline Crystal direction & $\begin{array}{c}\mathrm{a} \\
\text { [D/unit cell] }\end{array}$ & $\begin{array}{c}\mathrm{b} \\
\text { [D/unit cell] }\end{array}$ & $\begin{array}{c}\mathrm{c} \\
\text { [D/unit cell] }\end{array}$ \\
\hline Ionic DM & 0 & 0 & 48.39 \\
Total electronic DM & 0.00075 & 0.00026 & 0.0881 \\
\hline
\end{tabular}


a)
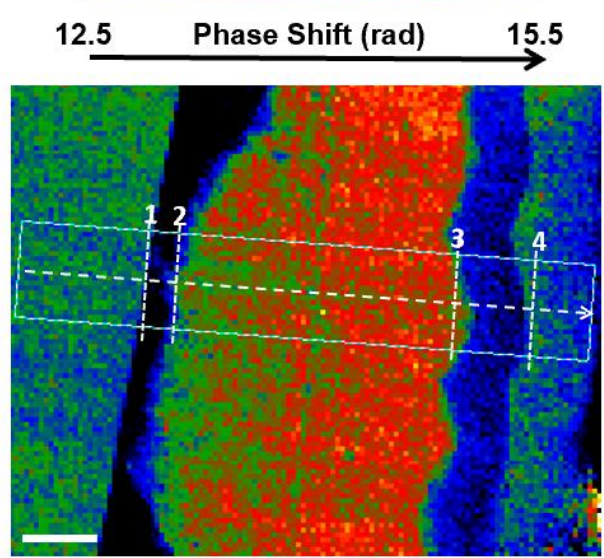

b)

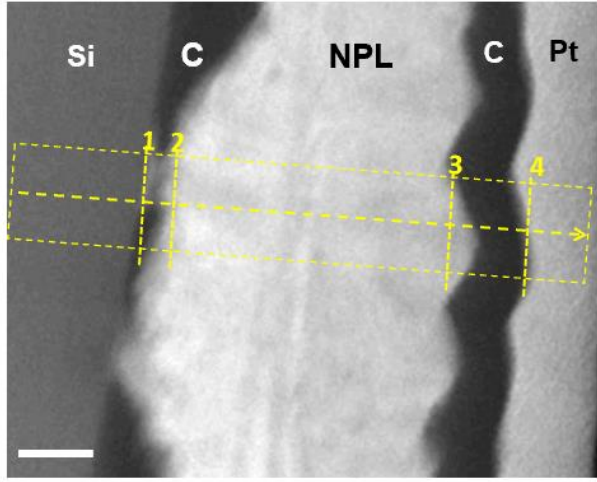

c)

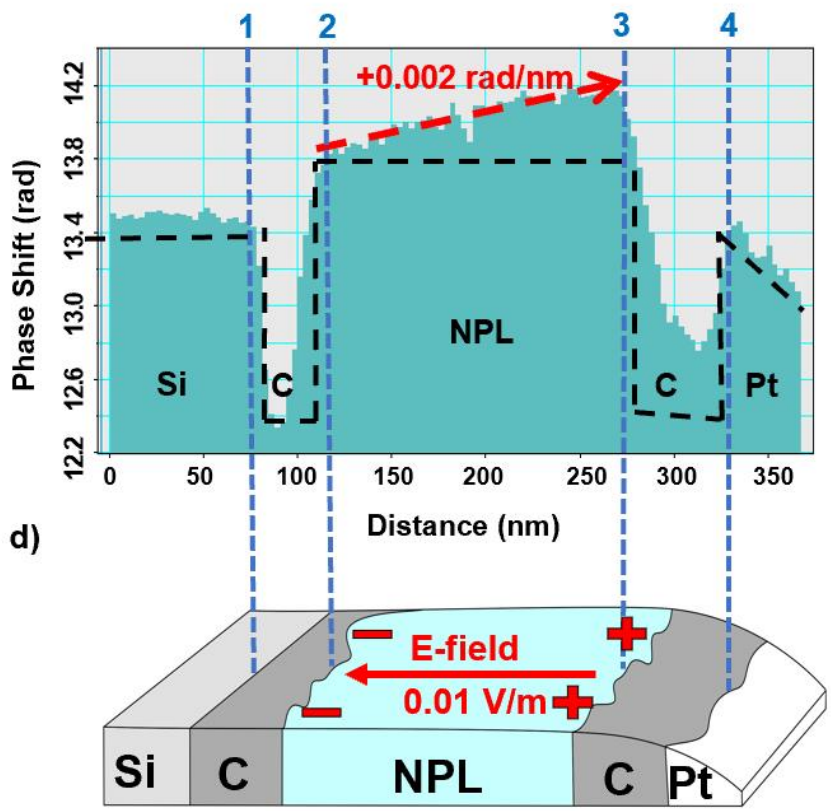

Figure 6. a) Electron holography phase-shift map of the cross-sectioned NPL. Note that the phase-shift slope in silicon was flattened $(0 \mathrm{rad} / \mathrm{nm})$ to compensate the slope from the mean inner potential contribution due to the tapered sample thickness variation (Supplementary figure 12). b) HAADF image of the same sample (scale bar $=50 \mathrm{~nm}$ ) c) The line phase profile with through-thickness direction (along the white dashed arrow in Fig. 5a). Black dashed line represents the phase-shift from the case where NPL has no electric field (only mean inner potential contributes to the phase-shift). The positive phase shift gradient (red dashed arrow, $\sim 0.02 \mathrm{rad} / \mathrm{nm}$ ) shown in experimental data for pure NPLs region represents the electrostatic asymmetry along the short axes of NPLs. d) Schematic description of the sample geometry and electric field pointing from the $\mathrm{Zn} / \mathrm{Cd}$-terminated plane (the line 3 , positive charge) to the $\mathrm{S}$ terminated plane (the line 2, negative charge). It should be noted that the negative slope in the Pt region is due to the rounded sample edge, which matches with estimated mean inner potential contribution. However, the positive slope in the NPL is opposite to the thickness variation, which attributes to the charge potential contribution. 


\section{Figures}
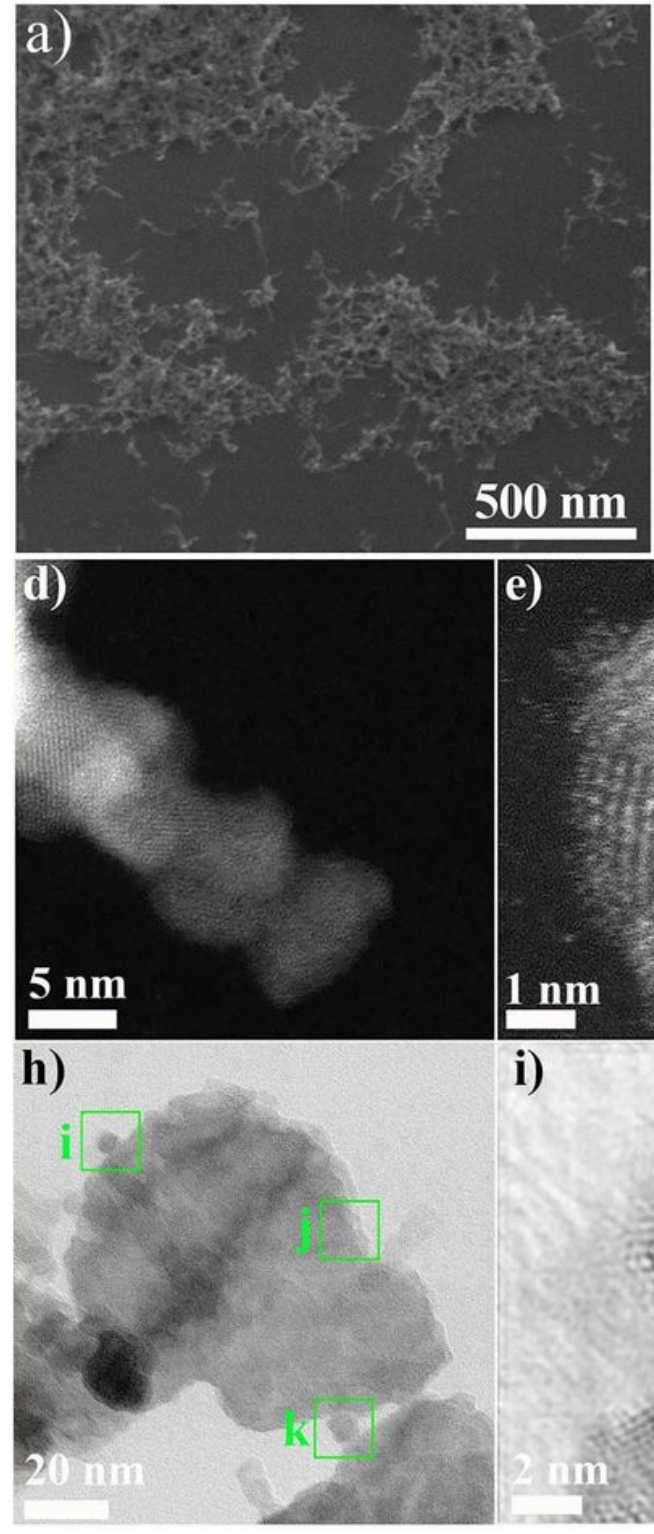
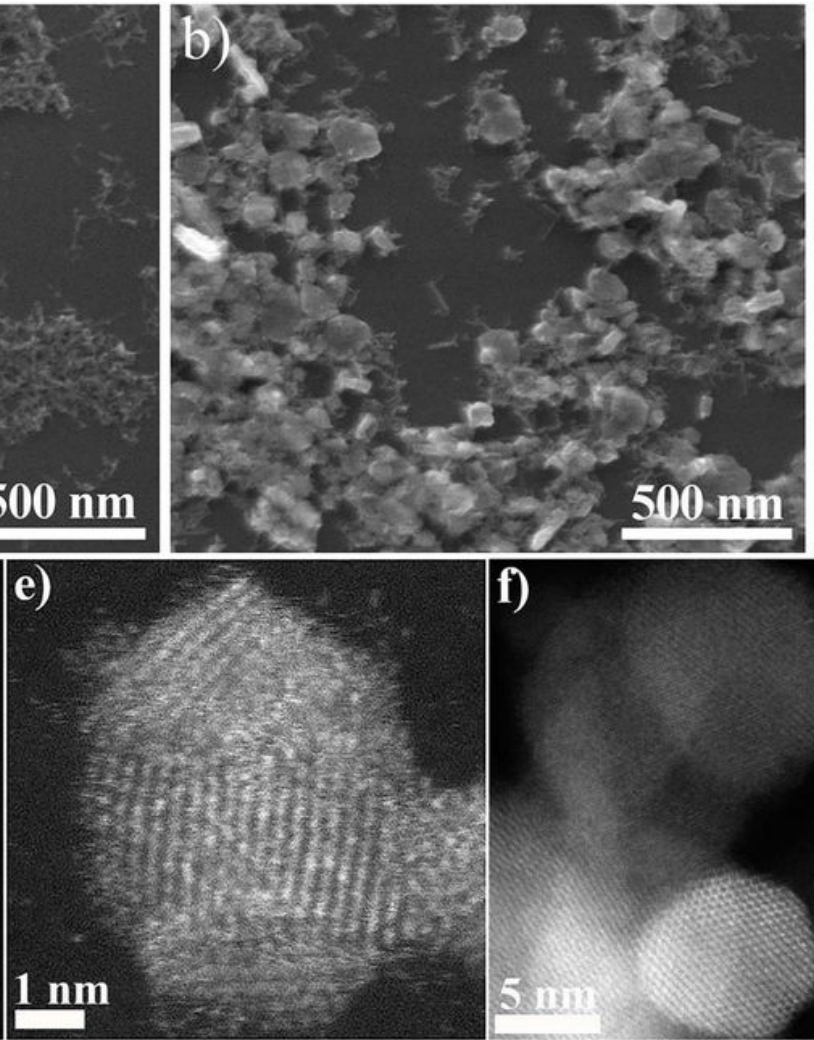

i)
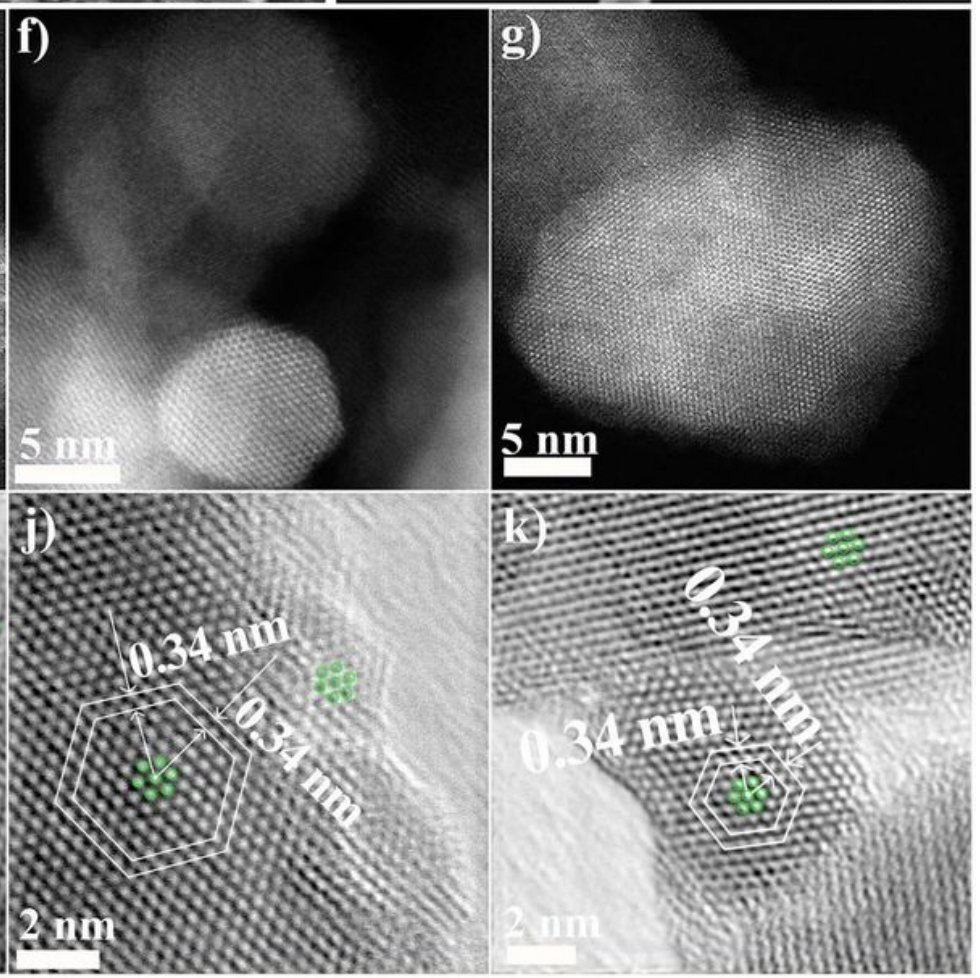

Figure 1

SEM images of the ZCS nanomaterial prepared at: a) $160^{\circ} \mathrm{C}$ for $48 \mathrm{~h}$; b) $220^{\circ} \mathrm{C}$ for $48 \mathrm{~h}$; c) Pure NPLs are obtained after purification. STEM ADF (d-g) and ( $h-k)$ BF images of the ZCS prepared at $220^{\circ} \mathrm{C}$ for $48 \mathrm{~h}$. 

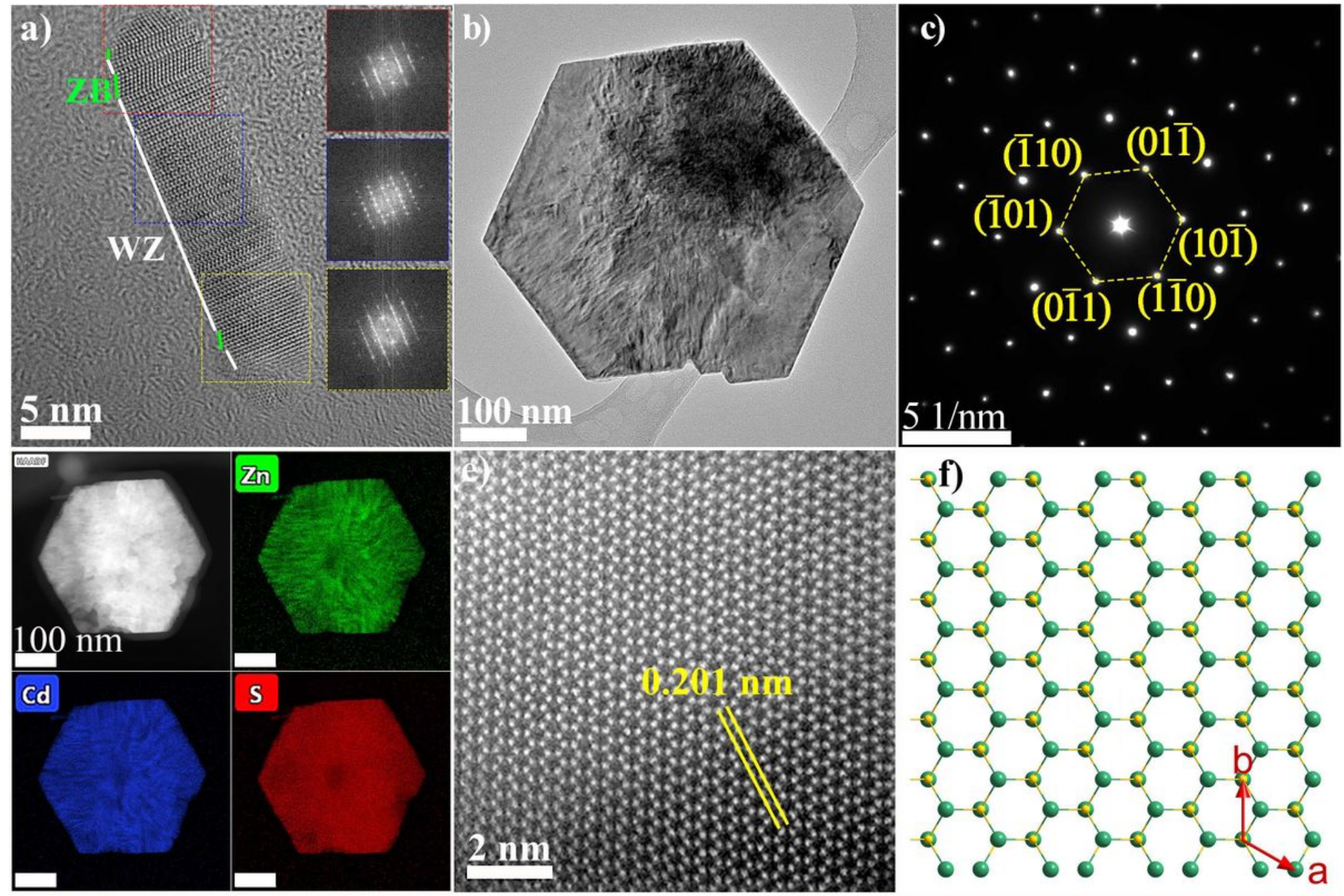

Figure 2

a) HRTEM images and FFT patterns of the ZCS NRs; b) TEM BF images of a ZCS NPLs; c) SAED pattern from the NPL in b); d) elemental mapping of the NPLs; e) Atomic resolution HAADF-STEM image along the [001] axis the NPLs. f) Atomic structure of the (001) plane of WZ-ZCS (green indicates Cd/Zn atom, yellow indicates $S$ atom). 

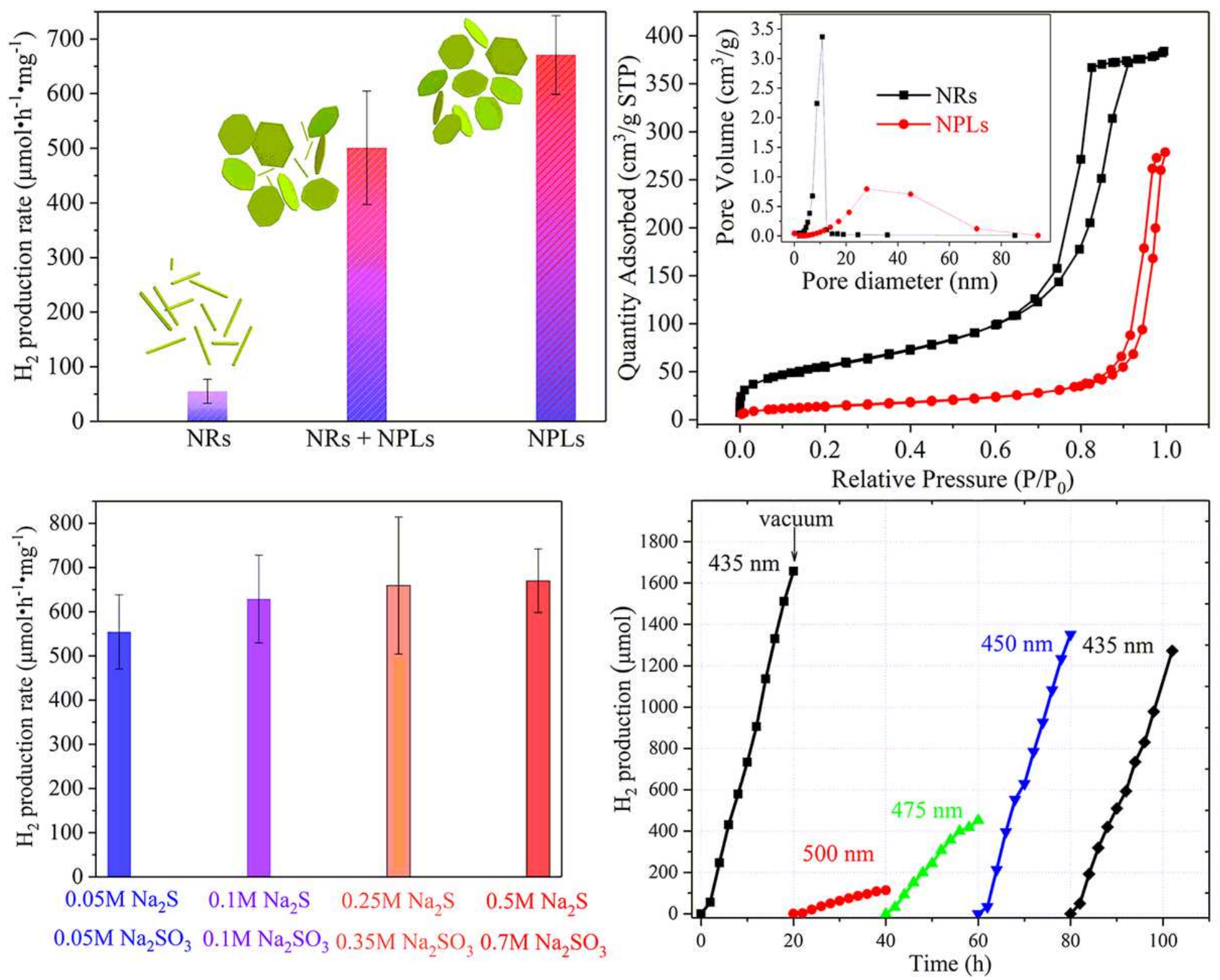

Figure 3

a) Photocatalytic $\mathrm{H} 2$ production rate for ZCS catalysts with different geometry: $1.0 \mathrm{mg}$ of photocatalyst, 0.7 M Na2S, and 1.05 M Na2SO3; b) Brunauer-Emmett-Teller adsorption-desorption isotherms of the NRs and the NPLs, pore size distribution by BJH in the insert; c) H2 production rate of the NPLs at different concentrations of hole scavengers. d) Cycling tests of photocatalytic activity of the NPLs under different wavelengths. Error bars in (b) and (c) represent standard deviation of measured rates within 3 hours. 
a)

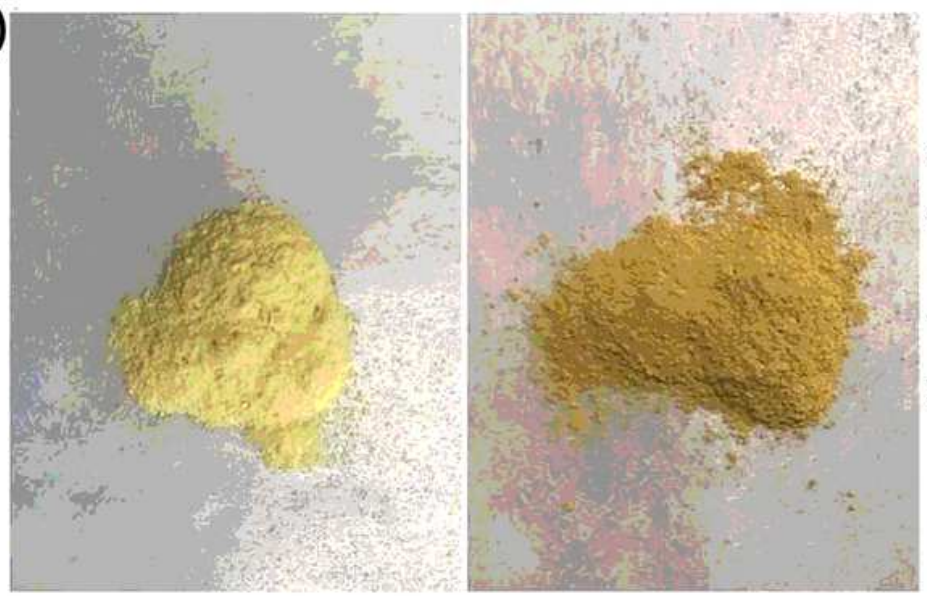

NRs
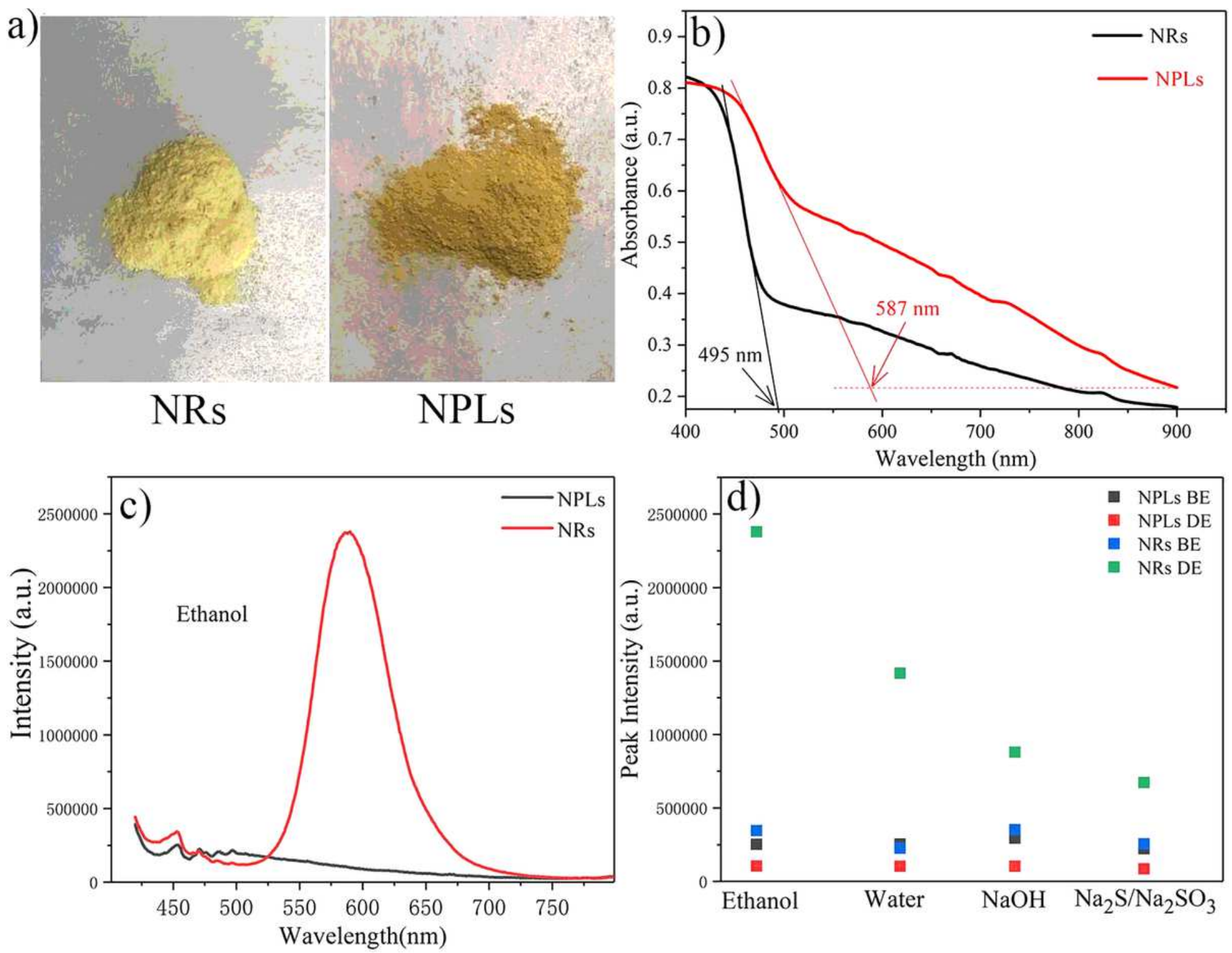

Figure 4

a) Photographs of the NRs and the NPLs samples used in photocatalysis experiments; b) UV-Vis spectra of the NRs and the NPLs, the inserts is the corresponding plots of (ahv)2 versus the energy of light (hv); Mott-Schottky plots of the NRs (c) and NPLs (d); Ultraviolet photoelectron spectra of the NRs (e) and NPLs (f); g)Photoluminescence spectra of NPLs and NRs (1 mg/mL) in ethanol; $h$ ) emission intensities in in ethanol, water, $\mathrm{NaOH}(10 \mathrm{M})$, and $\mathrm{Na} 2 \mathrm{~S}(0.7 \mathrm{M}) / \mathrm{Na} 2 \mathrm{SO} 3(1.05 \mathrm{M})$ solutions. The excitation wavelength was $400 \mathrm{~nm}$. 

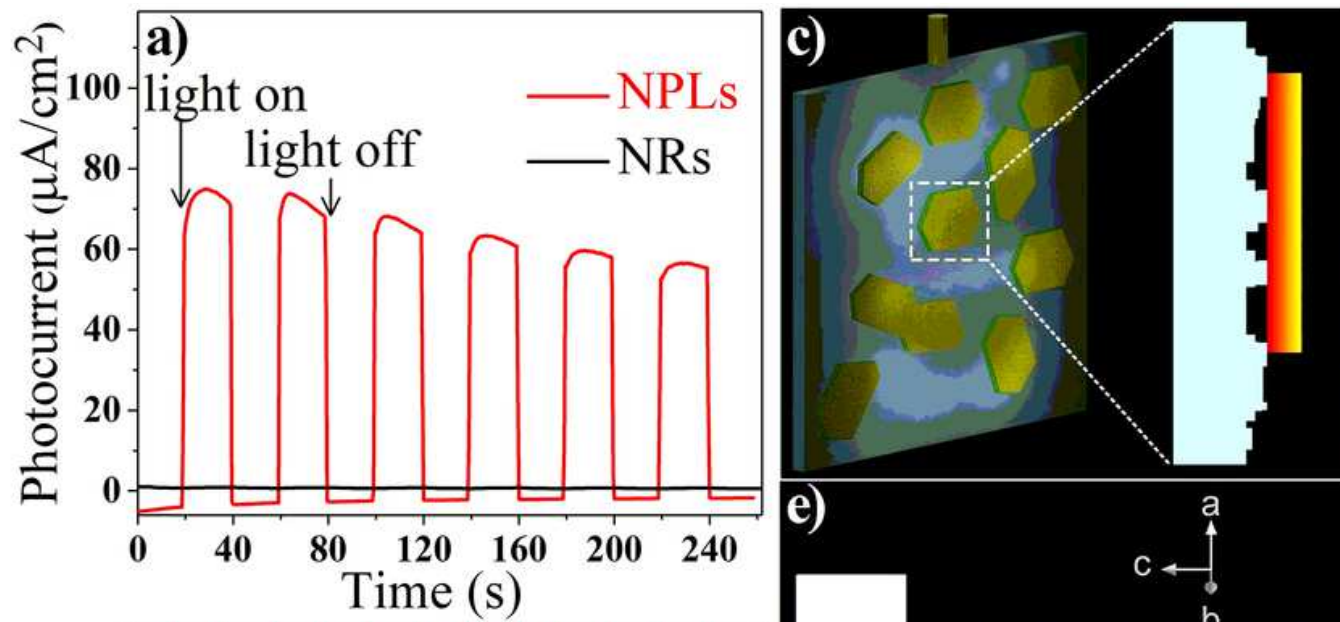

d)
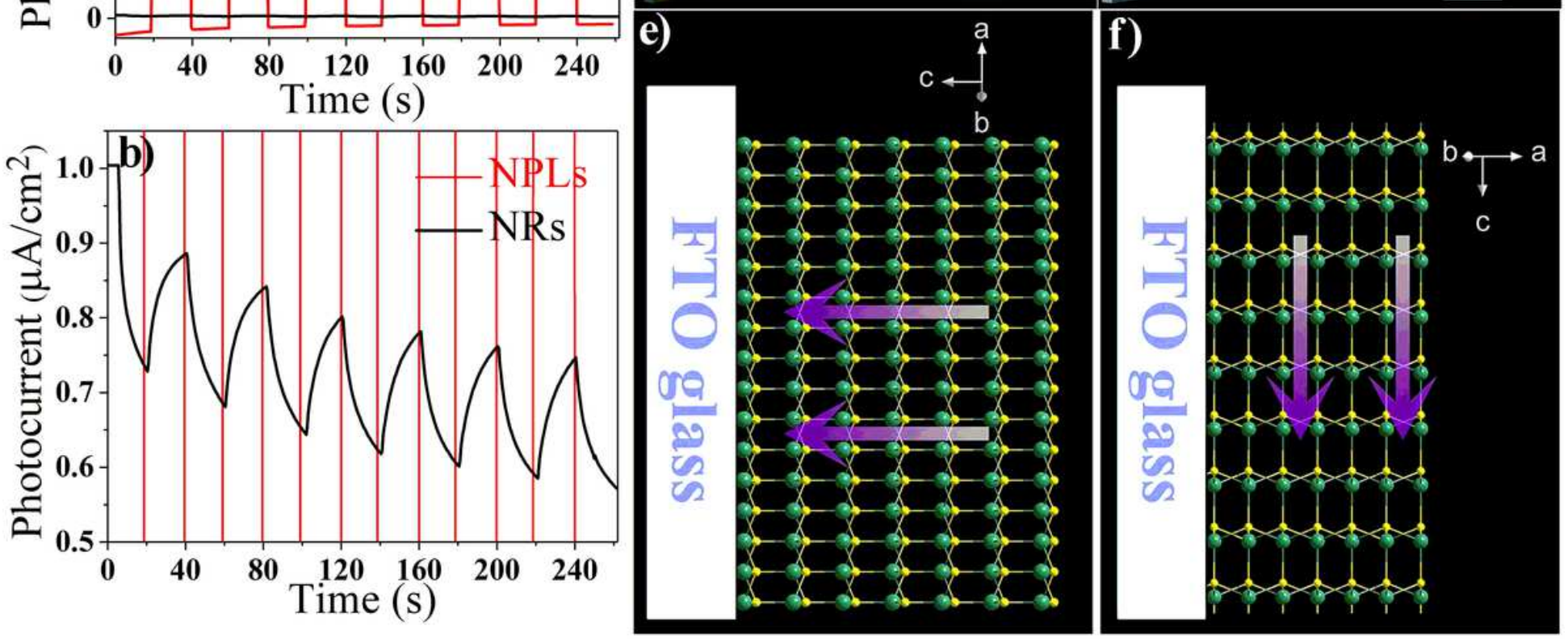

Figure 5

Photocurrent response (a) and locally enlarged photocurrent response (b) of the NRs and the NPLs. Schematic illustration of working electrode: (c) the NPLs and (d) the NRs, the inserts show the side view of electrodes. The color gradient indicates the charge gradient in the nanostructure. Schematic illustration of the intrinsic polarization field along the [001] direction for (e) the NPLs and (f) the NRs adsorbed to an FTO electrode. 
a)

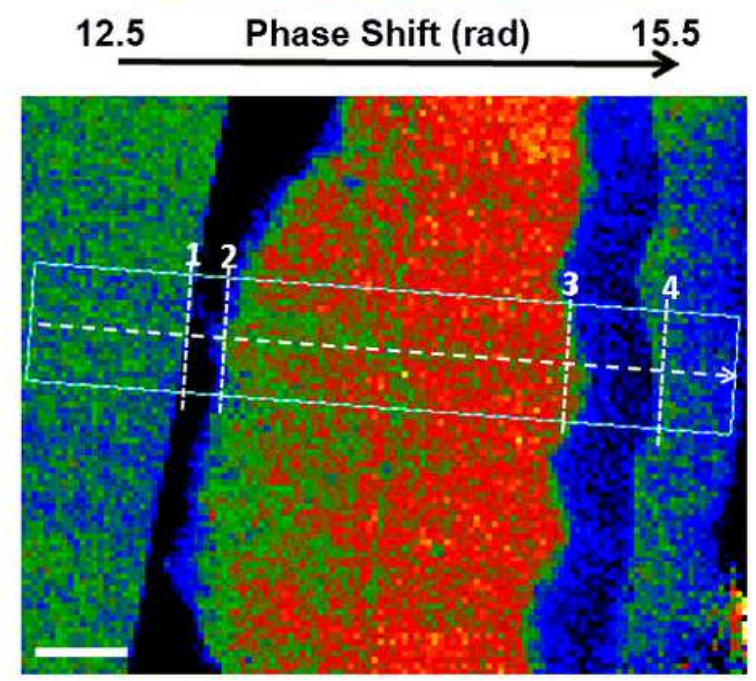

b)

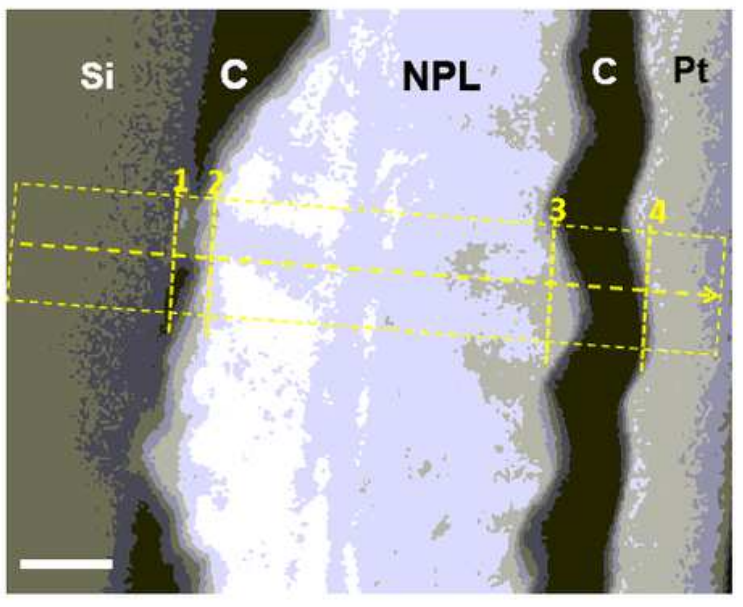

c)

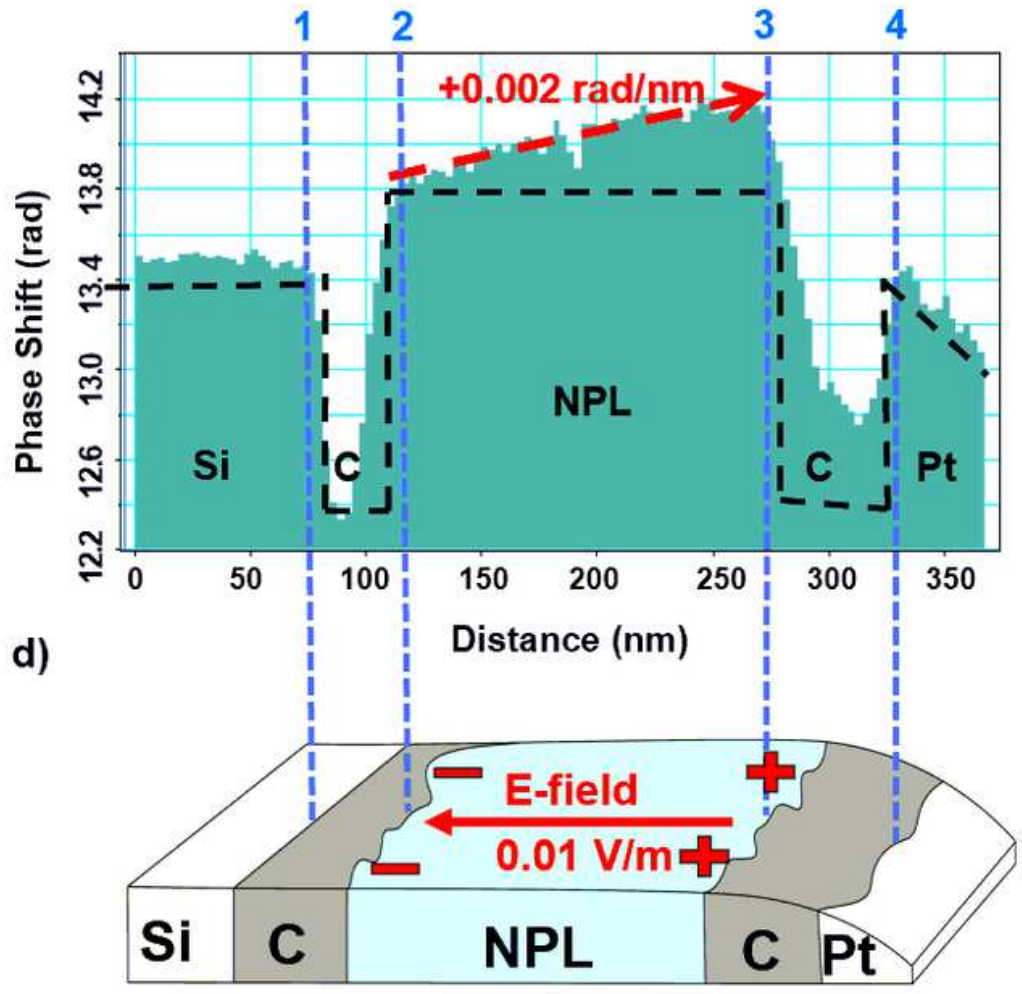

Figure 6

a) Electron holography phase-shift map of the cross-sectioned NPL. Note that the phase-shift slope in silicon was flattened ( $0 \mathrm{rad} / \mathrm{nm}$ ) to compensate the slope from the mean inner potential contribution due to the tapered sample thickness variation (Supplementary figure 12). b) HAADF image of the same sample (scale bar $=50 \mathrm{~nm}$ ) c) The line phase profile with through-thickness direction (along the white dashed arrow in Fig. 5a). Black dashed line represents the phase-shift from the case where NPL has no electric field (only mean inner potential contributes to the phase-shift). The positive phase shift gradient (red dashed arrow, $0.02 \mathrm{rad} / \mathrm{nm}$ ) shown in experimental data for pure NPLs region represents the electrostatic asymmetry along the short axes of NPLs. d) Schematic description of the sample geometry and electric field pointing from the $\mathrm{Zn} / \mathrm{Cd}$-terminated plane (the line 3, positive charge) to the Sterminated plane (the line 2 , negative charge). It should be noted that the negative slope in the Pt region is due to the rounded sample edge, which matches with estimated mean inner potential contribution. However, the positive slope in the NPL is opposite to the thickness variation, which attributes to the charge potential contribution. 


\section{Supplementary Files}

This is a list of supplementary files associated with this preprint. Click to download.

- movieS1.mov

- SIElectrostaticAsymmetryofNanoparticles.pdf 